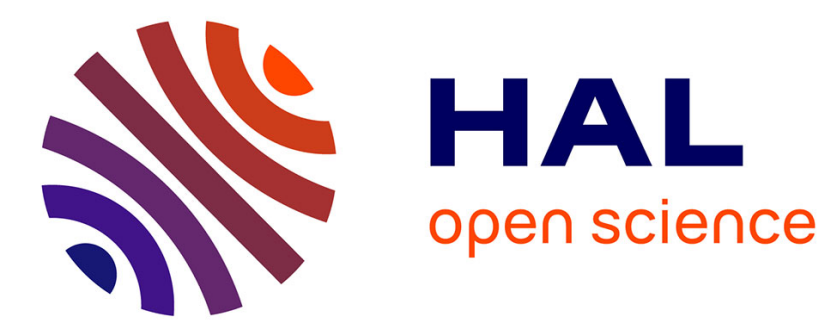

\title{
Affine recourse for the robust network design problem: Between static and dynamic routing
}

\author{
Michael Poss, Christian Raack
}

\section{To cite this version:}

Michael Poss, Christian Raack. Affine recourse for the robust network design problem: Between static and dynamic routing. Networks, 2013, 61 (2), pp.180-198. 10.1002/net.21482 . hal-00916978

\section{HAL Id: hal-00916978 \\ https://hal.science/hal-00916978}

Submitted on 11 Dec 2013

HAL is a multi-disciplinary open access archive for the deposit and dissemination of scientific research documents, whether they are published or not. The documents may come from teaching and research institutions in France or abroad, or from public or private research centers.
L'archive ouverte pluridisciplinaire HAL, est destinée au dépôt et à la diffusion de documents scientifiques de niveau recherche, publiés ou non, émanant des établissements d'enseignement et de recherche français ou étrangers, des laboratoires publics ou privés. 


\title{
Affine recourse for the robust network design problem: between static and dynamic routing
}

\author{
Michael Poss * $\quad$ Christian Raack ${ }^{\dagger}$
}

\begin{abstract}
Affinely-Adjustable Robust Counterparts provide tractable alternatives to (two-stage) robust programs with arbitrary recourse. Following Ouorou and Vial, we apply them to robust network design with polyhedral demand uncertainty, introducing the notion of affine routing. We compare the new affine routing scheme to the well-studied static and dynamic routing schemes for robust network design. It is shown that affine routing can be seen as a generalization of the widely-used static routing while still being tractable and providing cheaper solutions. We investigate properties of the demand polytope under which affine routings reduce to static routings and also develop conditions on the uncertainty set leading to dynamic routings being affine. We show however that affine routings suffer from the drawback that (even totally) dominated demand vectors are not necessarily supported by affine solutions. Uncertainty sets have to be designed accordingly. Finally, we present computational results on networks from SNDlib. We conclude that for these instances the optimal solutions based on affine routings tend to be as cheap as optimal network designs for dynamic routings. In this respect the affine routing principle can be used to approximate the cost for two-stage solutions with free recourse which are hard to compute.
\end{abstract}

Keywords: Robust optimization; Network design; Recourse; Affine Adjustable Robust Counterparts; Affine Routing; Demand polytope.

\section{Introduction}

In the classical deterministic network design problem, a set of point-to-point commodities with known demand values is given, and capacities have to be installed on the network links at minimum

*CMUC, Department of Mathematics, University of Coimbra, 3001-454 Coimbra, Portugal; GOM, Department of Computer Science, Faculté des Sciences, Université Libre de Bruxelles, Brussels, Belgium, mposs@ulb.ac.be. Michael Poss has been supported by CMUC and FCT (Portugal), through European program COMPETE/ FEDER within project PEst-C/MAT/UI0324/2011 and under the postdoctoral scholarship SFRH/BPD/76331/2011.

†Zuse Institute Berlin (ZIB), Takustr. 7, D-14195 Berlin, Germany, raack@zib.de. Christian Raack has been supported by the DFG research Center MAtheon, Berlin, Germany. 
cost such that the resulting capacitated network is able to accommodate all demands simultaneously by a multi-commodity flow. When demands are known with precision, this problem has been studied extensively in the literature, involving decompositions algorithms, extended formulations and strong cutting planes; see [6, 19, 24, 25, 28, 47, among others.

In practice, however, exact demand values are usually not known at the time the design decisions must be made. In telecommunications, demands are estimated which can be done for instance by using traffic measurements [51] or population statistics [20, 27]. These estimations allow the problem to be formulated and solved using classical tools of deterministic mathematical programming. However, the actual traffic forecast is strongly overestimated in order to yield robust networks capable of routing potential traffic peaks. Such overestimation results in over-provisioned networks wasting capital as well as operational expenditures such as energy resources.

By introducing so-called uncertainty sets, robust optimization overcomes this problem by explicitly taking into account the uncertainty of the data. A solution is said to be feasible if it is feasible for all realizations of the data in a predetermined uncertainty set $\mathcal{D}[13,14,18,50$. The original framework of Soyster [50] assumes that all decisions must be identical for all values of the uncertain parameters. Introducing more flexibility, two-stage robust optimization allows an adjustment of a subset of the problem variables only after observing the actual realization of the data [11. In fact, it is natural to apply this two-stage approach to network design since very often (first stage) capacity design decisions are made in the long term while the actual (second stage) routing is adjusted based on observed user demands. This second stage adjusting procedure is called recourse which in the context of (telecommunications) network design relates to what is known as traffic engineering. Unrestricted second stage recourse in robust network design is called dynamic routing [22, 31, 38. Given a fixed design, the commodity routing can be changed arbitrarily for every realization of the demands. Chekuri et al. 22] and Gupta et al. 31] show that allowing for dynamic routing makes robust network design intractable. More precisely, it is coNP-complete to decide whether a capacitated network allows for a dynamic routing of demands when the uncertainty set $\mathcal{D}$ of all possible demand vectors is a polytope. This is true even if $\mathcal{D}$ is described by polynomial many inequalities. We refer to Mattia [38] on how to handle the hardness of the separation problem when solving robust network design problems with dynamic routing. Mattia also provides a computational comparison to the static version.

Even more general, Ben-Tal et al. 11] observe that two-stage robust linear programming with free recourse is computationally intractable and suggest limiting the flexibility in the second stage by affine functions which makes the problem tractable. Chen and Zhang [23] extend this idea by using extended formulations of uncertainty sets and by applying affine recourse in the resulting higher-dimensional variable spaces.

Interestingly, this limitation in the flexibility of the second stage recourse has been used earlier 
in robust network design without relating it to two-stage optimization. Ben-Ameur and Kerivin [9, 10] introduce the concept of static routing which is also known as oblivious routing. After fixing the design, the routing of a commodity is allowed to change but the actual flow has to be a linear function of the observed demand of the same commodity. Static routing results in a fixed set of paths for every commodity and also a fixed percentage splitting of flow among these paths independent of the realization of the commodity demand. In this context we speak of a routing template used by all demand realizations. Robust network design with static routing can be handled as a single-stage problem introducing template variables; see the next section. Static routing has been used extensively since then. Ordóñez and Zhao [41] study structural properties of the robust network design problem when cost and demand values belong to conic uncertainty sets. Altin et al. 1] develop a compact integer linear programming model for virtual private network design with continuous capacities and single path routing. Altin et al. [2, 3] and Koster et al. [35, 36] study the network design problem assuming splittable flow and integer capacities. Polyhedral investigations and computational evaluations of the models are carried out.

The restriction of routing templates for every commodity makes the problem tractable but it is of course very conservative in terms of capacity cost compared to dynamic routing. Recently there have been several attempts to handle less restrictive routing principles. These could be shown to be intractable just like the dynamic case. Ben-Ameur $[8$ partitions the demand uncertainty set into two (or more) subsets using hyperplanes and devises specific routings for each subset. The resulting optimization problem is NP-hard when no assumptions are made on the hyperplanes. Ben-Ameur thus discusses simplifications where either the entire hyperplane or its direction is given. Scutellà [48, 49] introduces a generalization of the procedure from [8] by allowing for two (or more) routing templates to be used conjointly and proves NP-hardness of the corresponding robust network design problem in [49]. Then she introduces an approximation that devises routing templates iteratively; that is, given a solution consisting of a routing template and a network design, Scutellà 48. allows some commodities to use a second routing template in order to reduce the overall capacity allocation. Scutellà shows that the resulting minimization problem is convex and involves a subroutine whose solution can be approximated in polynomial time for every approximation factor.

As an alternative to these NP-hard approaches, Ouorou and Vial [45] and Babonneau et al. [7] only recently apply directly the affine recourse from [11] to network design problems using particular uncertainty sets. The resulting restrictive routing scheme is referred to as affine routing in the following. Affine routing can be seen as a generalization of static routing. In this context affine routing provides an alternative in between static and dynamic routing, yielding tractable robust counterparts in contrast to the schemes used in [8, 48].

The contribution of this paper consists of a theoretical and empirical study of network design 
under the affine routing principle for general polyhedral demand uncertainty sets $\mathcal{D}$. We embed affine routing into the context of two-stage network design with recourse and compare it to its natural counterparts, static and dynamic routings. Section 2 introduces the mathematical models and defines formally static, affine and dynamic routings. In Section 3 we show that, when $\mathcal{D}$ is fulldimensional, affine routings decompose into a combination of cycles and paths; that is, a routing template for every commodity can be affinely adjusted using a set of cycles, whenever a different commodity is perturbed within the feasible demand region. We describe then necessary and sufficient conditions on $\mathcal{D}$ under which affine routing is equivalent to static or dynamic routing. As a by-product, we obtain that static and dynamic routings are equivalent under certain assumptions on $\mathcal{D}$. We show that dominated demand vectors are not automatically supported by affine solutions in contrast to static and dynamic solutions. In particular very small demand scenarios have to be included in the uncertainty set. Finally, Section 4 presents numerical comparisons of dynamic, affine and static routings carried out on instances from SNDlib [43. It turns out that for these instances the affine routing principle is numerically very close to the dynamic second stage recourse rule. In fact, it provides enough flexibility to approximate the cost for optimal two-stage solutions with full flexibility.

An extended abstract of this paper has been published in [46].

\section{Robust network design with recourse}

We are given a directed graph $G=(V, A)$ and a set of commodities $K$. A commodity $k \in K$ has source $s(k) \in V$, destination $t(k) \in V$, and demand value $d^{k} \geq 0$. A flow for $k$ is a vector $f^{k} \in \mathbb{R}_{+}^{|A|}$ satisfying:

$$
\sum_{a \in \delta^{+}(v)} f_{a}^{k}-\sum_{a \in \delta^{-}(v)} f_{a}^{k}=d^{k} \psi_{v k} \quad \text { for all } v \in V
$$

where $\delta^{+}(v)$ and $\delta^{-}(v)$ denote the set of outgoing arcs and incoming arcs at node $v$, respectively. For node $v \in V$ and commodity $k \in K$ we set $\psi_{v k}:=1$ if $v=s(k), \psi_{v k}:=-1$ if $v=t(k)$, and $\psi_{v k}:=0$ otherwise. Flows are non-negative. A multi-commodity flow is a collection of flows, one for each commodity in $K$. A circulation (or cycle-flow) is a vector $g \in \mathbb{R}^{|A|}$ satisfying

$$
\sum_{a \in \delta^{+}(v)} g_{a}-\sum_{a \in \delta^{-}(v)} g_{a}=0 \quad \text { for all } v \in V .
$$

A circulation is not necessarily non-negative. A value $g_{a}<0$ can be seen as a flow from the head of arc $a$ to its tail. We call a circulation $g$ non-negative if $g \geq 0$ and positive if additionally $g \neq 0$. Notice that any two flows $\hat{f}^{k}, f^{k}$ for $k$ only differ by a circulation; that is, there always exists a circulation $g$ such that $\hat{f}^{k}=f^{k}+g$. 
In many practical situations, the demand vector $d \in \mathbb{R}_{+}^{|K|}$ is uncertain. In the following we assume that $d \in \mathcal{D} \subset \mathbb{R}_{+}^{|K|}$ where $\mathcal{D}$ is a bounded set. We often call $\mathcal{D}$ the uncertainty set. Any $d \in \mathcal{D}$ is said to be a realization of the demand. A routing is a function $f: \mathcal{D} \rightarrow \mathbb{R}_{+}^{|A| \times|K|}$ that assigns a multi-commodity flow to every realization of the demand. We say that $f$ serves $\mathcal{D}$ and call $f$ a dynamic routing if there is no further restriction on the routing. A capacity allocation $x \in \mathbb{R}_{+}^{|A|}$ is said to support the set $\mathcal{D}$ if there exists a routing $f$ serving $\mathcal{D}$ such that for every $d \in \mathcal{D}$ the corresponding multi-commodity flow $f(d)$ does not exceed the arc capacities given by $x$. Robust network design now aims at providing a capacity allocation supporting $\mathcal{D}$ at minimum cost. In this respect, robust network design is a two-stage robust program with recourse, following the more general framework described by [11]. The capacity design has to be fixed in the first stage, and, observing a demand realization $d \in \mathcal{D}$, we are allowed to adjust the routing $f(d)$ in the second stage. The problem can be written as the following (infinite) linear program denoted by $(R N D)$ in the following:

$$
\begin{aligned}
\min \sum_{a \in A} \kappa_{a} x_{a} & \\
(R N D) \quad \text { s.t. } \sum_{a \in \delta^{+}(v)} f_{a}^{k}(d)-\sum_{a \in \delta^{-}(v)} f_{a}^{k}(d) & =d^{k} \psi_{v k}, \quad v \in V, k \in K, d \in \mathcal{D} \\
\sum_{k \in K} f_{a}^{k}(d) & \leq x_{a}, \quad a \in A, d \in \mathcal{D} \\
f_{a}^{k}(d) & \geq 0, \quad a \in A, k \in K, d \in \mathcal{D} \\
x_{a} & \geq 0, \quad a \in A,
\end{aligned}
$$

where $\kappa_{a} \in \mathbb{R}_{+}$is the cost for installing one unit of capacity on arc $a \in A$. Ben-Tal et al. [11] point out that allowing for arbitrary recourse very often makes robust optimization problems intractable. In fact, this is true for two-stage robust network design with free recourse. It is known that deciding whether a given capacity vector $x$ supports $\mathcal{D}$ is coNP-complete for general polytopes $\mathcal{D}[22$, 31]. It follows from this hardness result that it is impossible (unless $\mathrm{P}=$ coNP) to derive a compact formulation for $(R N D)$ given a general uncertainty polytope $\mathcal{D}$ if there is no restriction on the second stage routing decision.

Most authors [2, 4, 10, 35, 36, 39, 41, use a simpler version of $(R N D)$ introducing a restriction on the second stage recourse known as static routing (also called oblivious routing). Each component $f^{k}: \mathcal{D} \rightarrow \mathbb{R}_{+}^{|A|}$ is forced to be a linear function of $d^{k}$ :

$$
f_{a}^{k}(d):=y_{a}^{k} d^{k} \quad a \in A, k \in K, d \in \mathcal{D}
$$

Notice that by (7) the flow for $k$ does not change if we perturb the demand for $h \neq k$. By combining (4) and (7) it follows that the multipliers $y \in \mathbb{R}_{+}^{|A| \times|K|}$ define a multi-commodity percentage flow; that is, for every $k \in K$, the vector $y^{k} \in \mathbb{R}_{+}^{|A|}$ satisfies (1) setting $d^{k}=1$. The flow $y$ is called a 
routing template since it decides, for every commodity, which paths are used to route the demand and what is the percentage splitting among these paths. The routing template has to be used by all demand scenarios $d \in \mathcal{D}$ under the static routing scheme.

Ben-Tal et al. [1] introduce Affine Adjustable Robust Counterparts restricting the recourse to be an affine function of the uncertainties. Applying this framework to $(R N D)$ means restricting $f^{k}$ to be an affine function of all components of $d$ giving

$$
f_{a}^{k}(d):=f_{a}^{0 k}+\sum_{h \in K} y_{a}^{k h} d^{h} \geq 0, \quad a \in A, k \in K, d \in \mathcal{D},
$$

where $f_{a}^{0 k}, y_{a}^{k h} \in \mathbb{R}$ for all $a \in A, k, h \in K$; see also [45. In what follows, a routing $f$ serving $\mathcal{D}$ and satisfying (8) for some vectors $f^{0}$ and $y$ is called an affine routing.

The following lemma formalizes the relation between optimal solutions of robust network design using dynamic, affine, or static routings. Affine routing generalizes static routing allowing for more flexibility in reacting to demand fluctuations, but it is not as flexible as dynamic routing.

Lemma 1. Let opt $t_{d y n}$, opt $t_{a f f}$, and opt $t_{\text {stat }}$ be the cost of the optimal solution to $(R N D)$ where $f$ is allowed to be dynamic, affine, or static, respectively. Then

$$
o p t_{d y n} \leq o p t_{a f f} \leq o p t_{\text {stat }} .
$$

Proof. Trivially any routing $f$ is a dynamic routing. The number of possible routings serving $\mathcal{D}$ is restricted by imposing (8) hence $o p t_{d y n} \leq o p t_{a f f}$. Moreover, we see immediately that static routing can be obtained from (8) by setting $f_{a}^{0 k}=0$ and $y_{a}^{k h}=0$ for each $a \in A$ and all $k, h \in K$ with $k \neq h$ yielding opt $t_{\text {aff }} \leq o p t_{\text {stat }}$.

Note that there is a proven worst-case optimality gap of $O(\log |V|)$ between the dynamic and static routing principle for undirected graphs [21. In fact this bound is tight. Goyal et al. 30] show that opt $_{d y n} \in O(|V|)$ while $o p t_{\text {stat }} \in \Omega(|V| \log |V|)$ for special graphs and using the polyhedral hose model. In this paper we do not establish optimality gaps between the three routing principles. We rather focus on studying properties of the demand scenarios $\mathcal{D}$ that either yield opt $_{\text {stat }}=$ opt $_{\text {aff }}$ or $o p t_{a f f}=o p t_{d y n}$. Our computational experiments in Section 4 suggest that for realistic networks and demand scenarios the static/dynamic optimality gap is small in practice (also see [38]), and if there is a gap, the cost for affine solutions tends to be very close to the cost for dynamic solutions. In fact in most cases opt $t_{a f f} \approx o p t_{d y n}$.

Given an uncertainty set $\mathcal{D}$, a static routing $f$ is completely described by the vector $y \in$ $\mathbb{R}_{+}^{|A| \times|K|}$. Similarly, an affine routing is completely described by fixing the vectors $f^{0} \in \mathbb{R}^{|A| \times|K|}$ and $y \in \mathbb{R}^{|A| \times|K| \times|K|}$. Extending the previous definitions, any routing template $y \in \mathbb{R}_{+}^{|A| \times|K|}$ is said to serve $\mathcal{D}$ if it yields a (static) routing $f$ serving $\mathcal{D}$. Similarly, any pair of vectors $f^{0} \in \mathbb{R}^{|A| \times|K|}$ and $y \in \mathbb{R}^{|A| \times|K| \times|K|}$ that satisfies (4) and (8) is said to serve $\mathcal{D}$. Given a capacity allocation 
$x \in \mathbb{R}_{+}^{|A|}$, the pair $(x, y)$ with $y$ serving $\mathcal{D}$ and the triplet $\left(x, f^{0}, y\right)$ with $\left(f^{0}, y\right)$ serving $\mathcal{D}$ are said to support $\mathcal{D}$ if the corresponding routings satisfy (5).

When $\mathcal{D}$ is not finite, model $(R N D)$ contains an infinite number of variables and inequalities. However, when $\mathcal{D}$ is a polytope, the following result shows that we can replace $\mathcal{D}$ by the set of its extreme points.

Lemma 2. Let $\mathcal{D} \subset \mathbb{R}_{+}^{|K|}$ be a bounded set. Further let $x \in \mathbb{R}^{|A|}$ be a capacity allocation, let $y \in \mathbb{R}_{+}^{|A| \times|K|}$ be a static routing serving $\mathcal{D}$, and let $\left(f^{0}, y^{\prime}\right) \in \mathbb{R}^{|A| \times|K|} \times \mathbb{R}^{|A| \times|K| \times|K|}$ be an affine routing serving $\mathcal{D}$. Then

(a) $x$ supports $\mathcal{D}$ if and only if $x$ supports $\operatorname{conv}(\mathcal{D})$.

(b) $(x, y)$ supports $\mathcal{D}$ if and only if $(x, y)$ supports $\operatorname{conv}(\mathcal{D})$.

(c) $\left(x, f^{0}, y^{\prime}\right)$ supports $\mathcal{D}$ if and only if $\left(x, f^{0}, y^{\prime}\right)$ supports $\operatorname{conv}(\mathcal{D})$.

Proof. $(c) \Leftarrow$ : Trivial.

$\Rightarrow$ : Consider $\left(x, f^{0}, y\right)$ that satisfies (4), (5) and (8) for each $d \in \mathcal{D}$. Any $d^{*} \in \operatorname{conv}(\mathcal{D})$ can be defined as follows: Let $d_{i} \in \mathcal{D}$ and $\lambda_{i} \geq 0, i=1, \ldots, n$ with $\sum_{i=1}^{n} \lambda_{i}=1$ and $d^{*}=\sum_{i=1}^{n} \lambda_{i} d_{i}$. In order to satisfy (8), $f\left(d^{*}\right)$ must be defined by

$$
f_{a}^{k}\left(d^{*}\right)=f_{a}^{0 k}+\sum_{h \in K} y_{a}^{k h} d^{h *}=\sum_{i=1}^{n} \lambda_{i}\left(f_{a}^{0 k}+\sum_{h \in K} y_{a}^{k h} d_{i}^{h}\right), \quad a \in A, k \in K, d \in \mathcal{D} .
$$

It is easy to see that $f_{a}^{k}\left(d^{*}\right)$ defined above together with $x$ satisfy (4) and (5). Statements $(a)$ and $(b)$ can be proved similarly.

When $\mathcal{D}$ is a polytope, Lemma 2 implies that $(R N D)$ can be discretized by restricting the model to the extreme demand scenarios that correspond to vertices of $\mathcal{D}$. This yields a linear programming reformulation of $(R N D)$ that has potentially an exponential number of variables and constraints. However, not all extreme points must be considered. For instance, if $0 \in \mathcal{D}$, it is an extreme point of $\mathcal{D}$ that any capacity allocation supports. This intuitive idea can be formalized using the domination concepts introduced by Oriolo [42]. Given two demands vectors $d_{1}$ and $d_{2}$, we say that $d_{1}$ dominates $d_{2}$ if any capacity allocation $x \in \mathbb{R}_{+}^{|A|}$ supporting $d_{1}$ also supports $d_{2}$. Moreover, $d_{1}$ totally dominates $d_{2}$ if any pair $(x, y)$ supporting $d_{1}$ also supports $d_{2}$. Thus, the optimal solution to $(R N D)$ with dynamic routing (resp. static routing) for a polytope $\mathcal{D}=\operatorname{conv}\left\{d_{1}, \ldots, d_{n}\right\}$ stays unchanged if we solve the problem without considering the dominated (resp. totally dominated) extreme points of $\mathcal{D}$. We refer to 42 for interesting characterizations of domination. We point out that the situation is different for affine routing. That is, we will show in this paper that it is impossible to define $d_{1}$ and $d_{2}, d_{1} \neq d_{2}$, such that any triplet $\left(x, f^{0}, y\right)$ supporting $d_{1}$ also supports $d_{2}$. 
In what follows, we will assume that $\mathcal{D}$ is a full-dimensional polytope, if not explicitly stated otherwise. Notice, however, that some results in Section 3 can be easily generalized to $\mathcal{D}$ being some bounded set in $\mathbb{R}_{+}^{|K|}$. We denote by $\operatorname{vert}(\mathcal{D})$ the set of extreme points of the polytope $\mathcal{D}$. The large linear program coming from the discretization of $\mathcal{D}$ can be sensibly simplified for affine and static routings. Namely, if $\mathcal{D}$ is defined by a polynomial number of inequalities, Ben-Tal and Nemirovski 12 show how to use the linear programming duality to obtain a formulation that contains the deterministic variables and constraints plus an additional polynomial number of variables and constraints, and that is equivalent to the robust semi-infinite program. Applegate and Cohen [5], among others, extend this idea to $(R N D)$ with static routing and we show in the next section how this can be done for $(R N D)$ with affine routing.

We close this section by pointing out some very trivial cases for which opt $t_{\text {stat }}=o p t_{\text {aff }}=o p t_{d y n}$. First, if $\mathcal{D}$ is a singleton (or even more generally if $\mathcal{D}$ contains a demand vector that totally dominates all $d \in \mathcal{D}$ ) then $o p t_{\text {stat }}=o p t_{a f f}=o p t_{d y n}$ since given any dynamic solution, the routing for the single (dominating) demand vector defines a static routing template at the same cost. Moreover, in this case every routing corresponding to an optimal solution is a shortest path routing; that is, the flow all commodities $k \in K$ is sent on a shortest path between $s(k)$ and $t(k)$ with respect to the arc capacity cost $\kappa_{a}$. To see this, observe that each capacity variable $x_{a}$ appears in a unique capacity constraint (because $\mathcal{D}$ is a singleton) so that $(R N D)$ decomposes into $|K|$ shortest path problems, one for every commodity. In such a case we speak of a shortest path template for $K$.

Secondly, in the single-commodity case, that is $|K|=1$, we obtain opt $t_{s t a t}=o p t_{a f f}=o p t_{d y n}$ since in this case the polytope $\mathcal{D} \subset \mathbb{R}_{+}^{|K|}$ is an interval, and we can solve the problem for the maximum single demand which dominates the interval. Also observe that (8) reduces to (7) in this case.

Finally, note that if there exists only one path from $s(k)$ to $t(k)$ for a commodity $k \in K$, then static, affine and dynamic routings coincide for that commodity. In the following, we assume that for all $k \in K$ there exist at least two distinct paths $p_{1}, p_{2}$ without cycles in $G$ from $s(k)$ to $t(k)$; that is, two paths that differ by at least one arc.

\section{Affine Routings}

In this section, we study properties and consequences of the affine routing principle. First, we remark that affine routing can be expressed as a routing template (just like in the static case) plus a set of circulations.

Lemma 3. Let $\left(f^{0}, y\right) \in \mathbb{R}^{|A| \times|K|} \times \mathbb{R}^{|A| \times|K| \times|K|}$ be an affine routing serving $\mathcal{D}$. Then $y^{k k} \in \mathbb{R}^{|A|}$ is a routing template for $k \in K$ and $f^{0 k} \in \mathbb{R}^{|A|}, y^{k h} \in \mathbb{R}^{|A|}$ are circulations for every $k, h \in K$ 
with $k \neq h$.

Proof. Plugging (8) into (4) and grouping together coefficients of each $d^{h}$, we obtain

$$
\begin{aligned}
\sum_{a \in \delta^{+}(v)} f_{a}^{0 k}-\sum_{a \in \delta^{-}(v)} f_{a}^{0 k} & +d^{k}\left(\sum_{a \in \delta^{+}(v)} y_{a}^{k k}-\sum_{a \in \delta^{-}(v)} y_{a}^{k k}\right) \\
& +\sum_{h \in K \backslash k} d^{h}\left(\sum_{a \in \delta^{+}(v)} y_{a}^{k h}-\sum_{a \in \delta^{-}(v)} y_{a}^{k h}\right)=d^{k} \psi_{v k}
\end{aligned}
$$

for each $v \in V, k \in K$. Let $e^{h}$ denote the unit vector in $\mathbb{R}^{|K|}$ corresponding to commodity $h$. Since $\mathcal{D}$ is full-dimensional there exists a vector $d_{0} \in \mathcal{D}$ and $\varepsilon>0$ such that $d_{0}+\varepsilon e^{h} \in \mathcal{D}$ for all $h \in K$. Subtracting (9) written for $d_{0}+\varepsilon e^{h}$ from (9) written for $d_{0}$ gives

$$
\varepsilon\left(\sum_{a \in \delta^{+}(v)} y_{a}^{k k}-\sum_{a \in \delta^{-}(v)} y_{a}^{k k}\right)=\varepsilon \psi_{v k}
$$

for $h=k$ and similarly

$$
\varepsilon\left(\sum_{a \in \delta^{+}(v)} y_{a}^{k h}-\sum_{a \in \delta^{-}(v)} y_{a}^{k h}\right)=0
$$

for $h \neq k$. Hence $y^{k k} \in \mathbb{R}^{|A|}$ is a routing template for $k \in K$ and $y^{k h} \in \mathbb{R}^{|A|}$ is a circulation if $k \neq h$. Plugging (10) and (11) into (9) also shows that $f^{0 k} \in \mathbb{R}^{|A|}$ is a circulation for all $k \in K$.

Just like in the static case, the flow for commodity $k$ changes linearly with $d^{k}$ on the paths described by the template $y_{a}^{k k}$. But in contrast to the static case, the flow for commodity $k$ may change also if the demand for $h \neq k$ changes which is described by circulations $y^{k h}$. However, the circulation flow $y^{k h}$ is proportional to $d^{h}$. In addition there is a constant circulation shift described by variables $f^{0 k}$.

As already mentioned, a dynamic routing for commodity $k$ could also be described by one (representative) routing template and circulations depending on the demand fluctuations. In the dynamic case the circulations can be chosen arbitrarily while in the affine case the actual flow changes according to $(8)$. We illustrate this concept in Example 1 and Figure 1 which shows that affine routing can be as good as dynamic routing in terms of the cost for capacity allocation. Example 1 also highlights that $f^{0}$ and $y^{k h}$ may not describe circulations when $\mathcal{D}$ is not fulldimensional

Example 1. Consider the network design problem for the graph in Figure 1(a) with two commodities $k_{1}: a \rightarrow b$ and $k_{2}: a \rightarrow c$. The uncertainty set $\mathcal{D}$ is defined by the extreme points $d_{1}=(2,1), d_{2}=(1,2)$, and $d_{3}=(1,1)$, and the capacity costs are the edge labels of Figure 1(a) Edge labels from Figure 1(b) and 1(c) represent optimal capacity allocations with static and dynamic routing, respectively. They have costs of 10 and 9, respectively. Then, Figure 1(d) $1(\mathrm{f})$ 


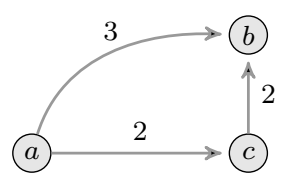

(a) edge costs

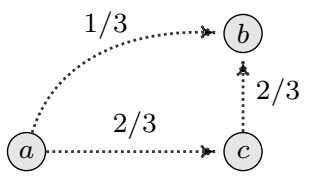

(d) $y^{k_{1} k_{1}}$

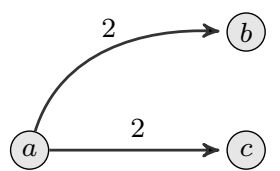

(b) static

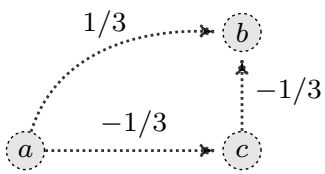

(e) $y^{k_{1} k_{2}}$

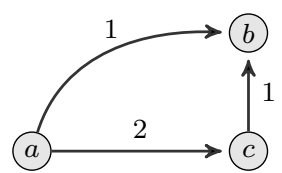

(c) dynamic

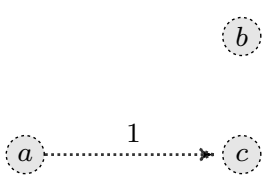

(f) $y^{k_{2} k_{2}}$

Figure 1: Optimal solutions for static, dynamic, and affine recourse.

describes coefficients $y^{k h}$ for an affine routing feasible for the capacity allocation 1(c). If we remove $d_{3}=(1,1)$ from the set of extreme points, the dimension of the uncertainty set reduces to 1 . The affine routing prescribed by $y_{a c}^{k_{2} k_{2}}=1, f_{a b}^{0 k_{1}}=3$, and $y_{a b}^{k_{1} k_{2}}=-1$ serves all demands in the convex hull of $d_{1}=(2,1)$ and $d_{2}=(1,2)$ but $f^{0 k_{1}}$ and $y^{k_{1} k_{2}}$ do not describe a circulation.

\subsection{A compact reformulation}

A formulation is said to be compact if the number of variables and constraints is polynomial in the number of nodes $|V|$. Because of Lemma 2, a compact linear formulation for the robust network design problem with affine routing can be prescribed whenever the number of (non-dominated) vertices of $\mathcal{D}$ is polynomial in the number of nodes, arcs, and commodities since we can restrict $(R N D)$ and (8) to these vertices. In the following we provide a compact linear reformulation for the case that $\mathcal{D}$ has a compact inequality description; that is, the polytope $\mathcal{D}$ has a linear description in $\mathbb{R}_{+}^{|K|}$ where the number of defining inequalities is polynomial in the number of nodes. The reformulation extends the one for static routing [4, 5]. Let $\mathcal{D}$ be given by

$$
\mathcal{D}:=\left\{d \in \mathbb{R}_{+}^{|K|}: \mathcal{A} d \leq b, d \geq 0\right\}
$$

where $\mathcal{A}=\left(\alpha^{i k}\right) \in \mathbb{R}^{m \times|K|}$ and $b \in \mathbb{R}^{m}, m \geq 1$.

Proposition 4. The robust network design problem $(R N D)$ (3)-(6) under the affine routing principle (8) respecting the uncertainty polytope $\mathcal{D}$ is equivalent to the following linear program denoted 
by $(A A R N D)$ :

$$
\begin{array}{rlrl}
\min \sum_{a \in A} \kappa_{a} x_{a} & & \\
\text { s.t. } \quad \sum_{a \in \delta^{+}(v)} y_{a}^{k k}-\sum_{a \in \delta^{-}(v)} y_{a}^{k k} & =\psi_{v k} & & v \in V, k \in K \\
\sum_{a \in \delta^{+}(v)} y_{a}^{k h}-\sum_{a \in \delta^{-}(v)} y_{a}^{k h} & =0 & & v \in V, k \neq h \in K \\
\sum_{a \in \delta^{+}(v)} f_{a}^{0 k}-\sum_{a \in \delta^{-}(v)} f_{a}^{0 k} & =0 & & v \in V, k \in K \\
\sum_{k \in K} f_{a}^{0 k}+\sum_{i=1}^{m} b_{i} \mu_{a}^{i} & \leq x_{a}, & & a \in A \\
\sum_{i=1}^{m} \alpha^{i h} \mu_{a}^{i} & \geq \sum_{k \in K} y_{a}^{k h}, & & a \in A, h \in K \\
\sum_{i=1}^{m} b_{i} \lambda_{a}^{i k} & \leq f_{a}^{0 k}, & & a \in A, k \in K \\
\sum_{i=1}^{m} \alpha^{i h} \lambda_{a}^{i k} & \geq-y_{a}^{k h}, & & a \in A, k \in K, h \in K \\
x, f^{0}, y, \mu, \lambda & \geq 0 &
\end{array}
$$

Proof. It has been shown in Lemma 3 that the flow balance constraints (4) reduce to (12)-(14) using (8). The capacity constraint (5) can be rewritten as

$$
\sum_{k \in K} f_{a}^{0 k}+\max _{d \in \mathcal{D}} \sum_{k \in K} \sum_{h \in K} d^{h} y_{a}^{k h} \leq x_{a}
$$

Dualizing the maximization problem in the constraint for every $a \in A$ using the inequality description of $\mathcal{D}$ gives 15 and $(16)$, where $\mu_{a}^{i}$ is the dual variable of the $i$-th inequality in $\mathcal{A} d \leq b$. The non-negativity constraints 8 can be rewritten as

$$
-f_{a}^{0 k}+\max _{d \in \mathcal{D}} \sum_{h \in K}-y_{a}^{k h} d^{h} \leq 0 .
$$

Dualizing the maximization problem in the constraint for every $a \in A$ and every $k \in K$ gives (17) and (18), where this time $\lambda_{a}^{i k}$ corresponds to the dual variable of the $i$-th inequality in the description of $\mathcal{D}$.

We note that reformulating by dualizing constraints has been proposed by [12] and has become a standard technique in robust linear optimization. Formulation $(A A R N D)$ generalizes the formulation of Ouorou and Vial [45] which considers affine routing for a specific polyhedral demand polytope $\mathcal{D}$. The model is obviously compact if $m$ is a polynomial function of $(|A|,|V|,|K|)$, that is, the description of $\mathcal{D}$ is compact. We remark that $\mathcal{D}$ being compact is not necessary to obtain a 


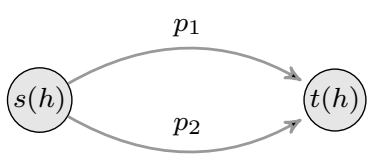

(a) paths

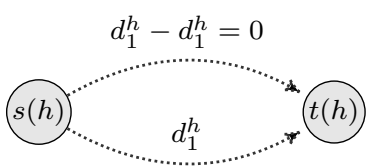

(b) resulting routing for $d_{1}$

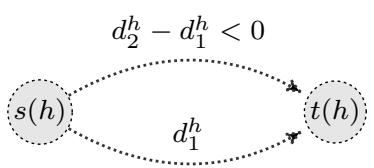

(c) resulting routing for $d_{2}$

Figure 2: Example of affine routing feasible for $d_{1}$ but not for $d_{2}$.

compact robust counterpart of $(R N D)$. It suffices to provide a compact extended formulation of $\mathcal{D}$ as shown in Section 4 . Since static routing is a special case of affine routing, formulation (12)-18 also generalizes the reformulation for static routing. The latter is obtained from $(12)-18$ by eliminating (13), 14 and (17) and fixing to zero the vectors $f^{0}, \lambda$ as well as the vectors $y^{k h}$ for all $k \neq h$.

Corollary 5. If either the number of vertices of $\mathcal{D}$ or its number of facets is polynomial in $|V|$, then $(R N D)$ with the affine recourse (8) can be solved in polynomial time.

Corollary 5 implies that given a capacity allocation $x$, the existence of an affine routing can be answered in polynomial time for every demand polytope with either polynomial many vertices or polynomial many facets contrasting with the hardness results using dynamic routing [22, 31].

\subsection{Domination of demand vectors}

The next result shows that it is not possible to define an "affine domination" between two demands vectors, in contrast to the domination and total domination defined by Oriolo [42] for dynamic and static routings, respectively.

Proposition 6. Let $d_{1}, d_{2} \in \mathbb{R}_{+}^{|K|}, d_{1} \neq d_{2}$. There exists $\left(x, f^{0}, y\right)$ that supports $d_{1}$ but not $d_{2}$.

Proof. Any $(x, y)$ that supports $d_{1}$ also supports $d_{2}$ if and only if $d_{1}^{k} \geq d_{2}^{k}$ for each $k \in K$ [42, Theorem 2.5]. Since static routings are special cases of affine routing we can assume that $d_{1}^{k} \geq d_{2}^{k}$ for each $k \in K$.

Since $d_{1} \neq d_{2}$, there exists $h \in K$ such that $d_{1}^{h}>d_{2}^{h}$. We describe next a capacity allocation and an affine routing that support $d_{1}$ but do not support $d_{2}$. Let $x_{a}=M$ for each $a \in A$ and $M$ large enough; for each $k \in K$, let $p_{k}$ be a path between $s(k)$ and $t(k)$ and let $y_{a}^{k k}=1$ for each $a \in p_{k}, 0$ otherwise. Then, let $y^{k_{1} k_{2}}=0$ for each $k_{1}, k_{2} \in K$ and $k_{1} \neq k_{2}$, and $f^{0 k}=0$ for each $k \in K \backslash\{h\}$. The construction for $h$ is illustrated in Figure 2, Let $p_{1}:=p_{h}$ and $p_{2}$ be a second path from $s(h)$ to $t(h)$ different from $p_{1}$; see Figure 2(a). Finally, set $f_{a}^{0 h}=-d_{1}^{h}$ for each $a \in p_{1} \backslash p_{2}$, $f_{a}^{0 h}=d_{1}^{h}$ for each $a \in p_{2} \backslash p_{1}$, and 0 otherwise. The defined affine routing shifts $d_{1}^{h}$ units of flow from path $p_{1}$ to path $p_{2}$ for commodity $h$. The triplet $\left(x, f^{0}, y\right)$ just defined supports $d_{1}$ with the 
flow depicted in Figure 2(b) but prescribes a negative flow on arcs $a \in p_{1} \backslash p_{2}$ for $d_{2}$ as depicted in Figure 2(c).

Given a demand polytope $\mathcal{D}$ for problem $(R N D)$ with dynamic (resp. static) routing, domination (resp. total domination) among demand vectors enables us to remove the dominated extreme points from $\mathcal{D}$, obtaining a smaller uncertainty set that is possibly easier to describe. For instance, 0 never needs to be considered. Proposition 6 shows that such simplification is not possible with affine routings.

Notice that it is possible to introduce an "affine domination" if $G$ is such that some commodities can use only one path. Namely, if for each $k \in Q \subseteq K$, there exists only one path from $s(k)$ to $t(k)$, then any triplet $\left(x, f^{0}, y\right)$ that supports $d_{1}$ also supports $d_{2}$ if and only if $d_{1}^{k}=d_{2}^{k}$ for $k \in K \backslash Q$ and $d_{1}^{k} \geq d_{2}^{k}$ otherwise.

Let us eventually comment on the consequences of Proposition 6 and the impact of affine routings. If the routing scheme is used in practice to provide more flexibility to a network facing uncertain demands, then Proposition 6 essentially shows that the uncertainty polytope can not be simplified by exploiting domination. In this sense, Proposition 6 is a negative result. Very often, however, the focus is not on the actual realized routing but on a feasible capacity design at low cost. In this context affine routing can be seen and used as an approximation scheme to $(R N D)$ with dynamic routing, the latter being very difficult computationally. Proposition 6 now tells us that removing the dominated extreme points from $\mathcal{D}$ may reduce the capacity cost for affine routings, and therefore, may yield a cheaper network that is still (dynamically) feasible and hence a better approximation to $(R N D)$ with free recourse. For this reason we consider two uncertainty sets for affine routing in our numerical experiments in Section 4 . The first handles the given demand polytope $\mathcal{D}$ while the second one removes dominated extreme points from $\mathcal{D}$.

\subsection{Relation to static routing}

The results from the previous section imply that all extreme points of $\mathcal{D}$ must be considered when using affine routing, in particular extreme points corresponding to very small demand realization. Also $0 \in \mathcal{D}$, if required, cannot be removed from the uncertainty set. This comes from the nonnegativity constraints 19 , which impose important restrictions on the circulation variables $f^{0 k}$ and $y^{k h}$. Unfortunately it turns out that affine routings reduce to static routings whenever $\mathcal{D}$ contains very small demand realization as we will show in the following. In what follows let $e^{k}$ be the $k$-th unit vector in $\mathbb{R}_{+}^{|K|}$.

Lemma 7. If $0 \in \mathcal{D}$ then $f^{0 k} \in \mathbb{R}_{+}^{|A|}$ describes a non-negative circulation in $G$. If $\epsilon e^{h} \in \mathcal{D}$ for some $\epsilon>0$ and $h \in K$ and $f^{0 k}=0$ for $h \neq k \in K$ then $y^{k h} \in \mathbb{R}_{+}^{|A|}$ describes a non-negative circulation in $G$. 


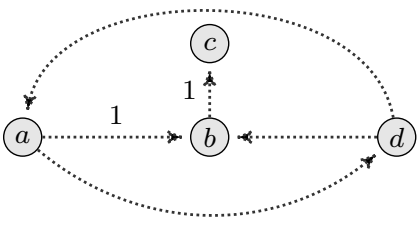

(a) $y^{k_{1} k_{1}}$

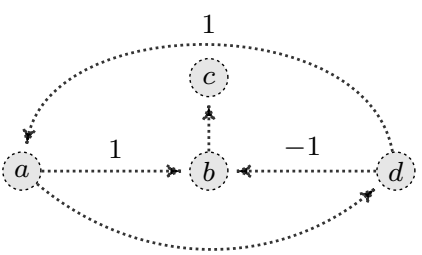

(b) $f^{0 k_{1}}$

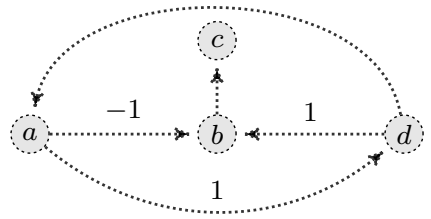

(c) $y^{k_{1} k_{2}}$

Figure 3: Irreducible affine routing for commodity $k_{1}$ that contains a positive circulation for all $d \in \mathcal{D}$.

Proof. Writing (8) for $0 \in \mathcal{D}$ gives $f_{a}^{0 k} \geq 0$ for all $a \in A$ and $k \in K$ and hence by Lemma $3 f^{0 k}$ is a non-negative circulation. Similarly, writing $(8)$ for $\epsilon e^{h} \in \mathcal{D}$ we get $f_{a}^{0 k}+\epsilon y_{a}^{k h} \geq 0$ for all $a \in A$ and $k \in K$. If $f^{0 k}=0$ for $k \neq h$ we get that $y^{k h}$ is a non-negative circulation by Lemma 3

It is clear that the non-negative circulations mentioned in Lemma 7 do not yield useful affine routings because they increase the capacity requirement without providing additional flexibility. If a flow $f^{k}$ for $k$ contains a positive circulation; that is, there exists a positive circulation $g$ such that $f^{k}-g$ is a flow for $k$, then $f^{k}$ can be reduced to $f^{k}-g$ without changing the flow balance at $s(k)$ and $t(k)$. Similarly, if a routing $f: \mathcal{D} \rightarrow \mathbb{R}_{+}^{|A| \times|K|}$ results in a positive circulation for some $d \in \mathcal{D}$ and $k \in K$ we can construct a second routing $\bar{f}$ serving $\mathcal{D}$ by removing this circulation. The routing $\bar{f}$ may require less capacity than $f$. However, if we suppose that $f$ is an affine routing, it is not guaranteed that the routing $\bar{f}$ constructed this way is also affine. In fact, we will see that in general this is not the case.

However, we can always remove positive circulations from the vectors $f^{0 k}$ and $y^{k h}, k, h \in K$ individually. Setting those components of $f^{0 k}$ and $y^{k h}$ to zero that correspond to a positive cycle-flow in $G$ does not change the flow balance constraints and the resulting affine routing is still serving $\mathcal{D}$ but requiring less capacity. In this spirit, we call an affine routing $f$ serving $\mathcal{D}$ irreducible if none of the defining flow and circulation vectors $f^{0 k}$ and $y^{k h}$ with $k, h \in K$ contains a positive circulation. Of course, every (affine) optimal solution corresponds to an affine routing that is irreducible. We mention that irreducible affine routings may still result in positive cycle-flows for all $d \in \mathcal{D}$, which we show in Figure 3 and the following Example 2

Example 2. Consider the digraph depicted in Figure 3 for the two commodities $k_{1}: a \rightarrow c$ and $k_{2}: a \rightarrow b$. The uncertainty set $\mathcal{D}$ is described by the extreme points $d_{1}=(1,2), d_{2}=(2,1)$, and $d_{3}=(1,1)$. Edge labels from Figure $3(\mathrm{a}) 3(\mathrm{c})$ represent the affine routing for $k_{1}$ and let the routing for $k_{2}$ be arbitrary. The affine routing just described is irreducible because neither $f^{0 k_{1}}$ nor $y^{k_{1} k_{2}}$ contains positive cycle-flows. However, this routing sends a flow of 1 along cycle $a \rightarrow e \rightarrow a$ for all $d \in \mathcal{D}$. Hence the resulting routing just described contains a positive cycle-flow for all $d \in \mathcal{D}$. 
In the case of irreducible affine routings, Lemma 7 provides conditions under which $f^{0 k}=0$ for $k \in K$ and $y^{k h}=0$ for all $k, h \in K, k \neq h$ and thus, the corresponding affine routing is static:

Proposition 8. If $0 \in \mathcal{D}$ and for each $k \in K$ there is $\epsilon_{k}>0$ such that $\epsilon_{k} e^{k} \in \mathcal{D}$, then all irreducible affine routings serving $\mathcal{D}$ are static and hence opt $t_{\text {aff }}=$ opt $_{\text {stat }}$.

Example 3. We mention that the hose polyhedron, often used to model data uncertainty in telecommunications networks [1, 2, 26, 29, usually fulfills the assumption of Proposition 8, Recall that the asymmetric version of this polyhedron is defined as

$$
\mathcal{D}^{H}=\left\{d \in \mathbb{R}_{+}^{|K|}: \sum_{k \in K: s(k)=v} d^{k} \leq b_{v}^{+} \text {and } \sum_{k \in K: t(k)=v} d^{k} \leq b_{v}^{-} \text {for all } v \in V\right\},
$$

for non-negative numbers $b_{v}^{+}$and $b_{v}^{-}$. If $b_{v}^{+}>0$ and $b_{v}^{-}>0$ for all $v \in V$, then $\mathcal{D}^{H}$ fulfills the assumptions of Proposition 8 so that all irreducible affine routings serving $\mathcal{D}^{H}$ are static and hence $o p t_{a f f}=o p t_{\text {stat }}$.

Proposition 8 also highlights that affine routing suffers from a drawback related to Proposition 6. Adding dominated or totally dominated vectors to $\mathcal{D}$ might restrict the set of feasible affine routings.

In the rest of this section, let $\mathcal{D}_{0}^{k}$ denote the set obtained from $\mathcal{D}$ by removing $d \in \mathcal{D}$ with $d^{k}>0$; that is, $\mathcal{D}_{0}^{k}:=\left\{d \in \mathcal{D}: d^{k}=0\right\}$. Whenever $G$ is acyclic, we show in the next proposition that the condition in Proposition 8 on the structure of $\mathcal{D}$ can be relaxed to $\operatorname{dim}\left(\mathcal{D}_{0}^{k}\right)=|K|-1$ for all $k \in K$. Moreover, we show below that $\operatorname{dim}\left(\mathcal{D}_{0}^{k}\right)=|K|-1$ for all $k \in K$ is a necessary and sufficient condition for static routings to be affine in acyclic graphs, while it is only a necessary condition for static routings to be affine in general graphs. Sufficiency and Necessity are shown in Proposition 9 and Theorem 10 , respectively. Notice that for acyclic graphs, all affine routings are irreducible.

Proposition 9. Let $G$ be an acyclic network. If $\operatorname{dim}\left(\mathcal{D}_{0}^{k}\right)=|K|-1$ for all $k \in K$, then all affine routings serving $\mathcal{D}$ are static and hence opt $t_{\text {aff }}=o p t_{\text {stat }}$.

Proof. Let $k \in K$ be a given commodity. For all $d \in \mathcal{D}_{0}^{k}$, any flow $f^{k}(d)$ must either be equal to 0 or describe a positive circulation. The latter is impossible because $G$ is acyclic, so that $f^{k}(d)=0$ for all $d \in \mathcal{D}_{0}^{k}$. Let $\left\{d_{1}, \ldots, d_{|K|}\right\}$ be a set of affinely independent vectors included in $\mathcal{D}_{0}^{k}$. Any affine flow for $k$ must satisfy

$$
f_{a}^{0 k}+\sum_{h \in K} y_{a}^{k h} d_{i}^{h}=f_{a}^{0 k}+\sum_{h \in K \backslash\{k\}} y_{a}^{k h} d_{i}^{h}=0, \quad 1 \leq i \leq|K|,
$$

for all $a \in A$, which is a system of $|K|$ independent linear equations with $|K|$ variables. Therefore, its unique solution is 0 and the affine routing must be static. 
In the following we prove that for general graphs the condition $\operatorname{dim}\left(\mathcal{D}_{0}^{k}\right)=|K|-1$ for all $k \in K$ is necessary for all irreducible affine routings to be static. This means that in all other cases there exists irreducible affine routings that are not static. But notice that from the latter it does not follow that opt $t_{\text {aff }}<o p t_{\text {stat }}$ as shown in Example 4.

Theorem 10. If all irreducible affine routings serving $\mathcal{D}$ are static then $\operatorname{dim}\left(\mathcal{D}_{0}^{k}\right)=|K|-1$ for all $k \in K$.

Proof. Let $k \in K$ be such that $\operatorname{dim}\left(\mathcal{D}_{0}^{k}\right)<|K|-1$. We construct an irreducible affine routing for commodity $k$ (all other commodities are routed arbitrarily). Consider two distinct paths $p_{1}$ and $p_{2}$ in $G$ from $s(k)$ to $t(k)$ that do not contain cycles. Let $y^{k k}$ be the routing template that splits the flow equally between $p_{1}$ and $p_{2}$; that is, $y_{a}^{k k}$ is equal to 0.5 for $a \in p_{1} \cup p_{2} \backslash\left(p_{1} \cap p_{2}\right), 1$ for $a \in p_{1} \cap p_{2}$, and 0 otherwise. We will construct an affine perturbation for $k$ using the cycles formed by $p_{2} \backslash p_{1}$ and $p_{1} \backslash p_{2}$ where arcs in $p_{1} \backslash p_{2}$ will be taken in the reverse direction (their flows will be negative). If $\mathcal{D}_{0}^{k}$ is non-empty, let $p-1$ be its dimension, with $1 \leq p \leq|K|-1$, and let $\left\{d_{1}, \ldots, d_{p}\right\}$ be a set of affinely independent vectors spanning $\mathcal{D}_{0}^{k}$. It follows that the system of equations

$$
\lambda^{0}+\sum_{h \in K \backslash k} d_{i}^{h} \lambda^{h}=0, \quad 1 \leq i \leq p
$$

has a solution $\bar{\lambda} \neq 0$. In case $\mathcal{D}_{0}^{k}$ is empty we choose the vector $\bar{\lambda} \neq 0$ arbitrarily. We construct next an affine perturbation for the flow of commodity $k$ based on $\bar{\lambda}$.

Given $\epsilon>0$, let $f_{a}^{0 k}=\epsilon \bar{\lambda}^{0}$ for $a \in p_{2} \backslash p_{1}, f_{a}^{0 k}=-\epsilon \bar{\lambda}^{0}$ for $a \in p_{1} \backslash p_{2}$, and $f_{a}^{0 k}=0$ otherwise. Similarly, given $h \neq k$, we set $y_{a}^{k h}=\epsilon \bar{\lambda}^{h}$ for all arcs $a$ in $p_{2} \backslash p_{1}, y_{a}^{k h}=-\epsilon \bar{\lambda}^{h}$ for $a \in p_{1} \backslash p_{2}$ and $y_{a}^{k h}=0$ otherwise. Combining these cycle variables with the routing template $y^{k k}$ defined above yields an irreducible affine routing $f^{k}$ serving $\mathcal{D}$.

It remains to show that $\epsilon>0$ can be chosen such that the flow $f^{k}(d)$ is non-negative on all $\operatorname{arcs}$ for all $d \in \mathcal{D}$. Because of Lemma 2 we can restrict ourselves to the finite $\operatorname{set} \operatorname{vert}(\mathcal{D})$ of extreme points of $\mathcal{D}$. Consider first $d \in \operatorname{vert}(\mathcal{D})$ with $d^{k}=0$. By definition, $f_{a}^{k}(d)=0$ for each $a \in A \backslash\left(p_{1} \cup p_{2}\right)$, and $f_{a}^{k}(d)=d^{k}=0$ for each $a \in p_{1} \cap p_{2}$. Assume $a \in p_{2} \backslash p_{1}$. The vector $d$ can be written as an affine combination of the vectors in $\left\{d_{1}, \ldots, d_{p}\right\}$; that is, $d=\sum_{i=1}^{p} \mu_{i} d_{i}$ for some multipliers $\mu_{i} \in \mathbb{R}$ with $\sum_{i=1}^{p} \mu_{i}=1$. Hence the flow on arc $a \in p_{2} \backslash p_{1}$ for demand $d$ satisfies

$$
f_{a}^{k}(d)=\epsilon\left(\bar{\lambda}^{0}+\sum_{h \in K \backslash k} d^{h} \bar{\lambda}^{h}\right)=\epsilon \sum_{i=1}^{p} \mu_{i}\left(\bar{\lambda}^{0}+\sum_{h \in K \backslash k} d_{i}^{h} \bar{\lambda}^{h}\right)=0,
$$

where the last equation follows from (22). Similarly, the flow can be shown to be zero for $a \in p_{1} \backslash p_{2}$.

Now let $d \in \operatorname{vert}(\mathcal{D})$ be such that $d^{k}>0$. Again $f_{a}^{k}(d)=0$ for $a \in A \backslash\left(p_{1} \cup p_{2}\right) \cup\left(p_{1} \cap p_{2}\right)$ by definition. For $a \in p_{2} \backslash p_{1}$ and $a \in p_{1} \backslash p_{2}$ it holds that $f_{a}^{k}(d)=0.5 d^{k}+\epsilon g(d)$ and $f_{a}^{k}(d)=$ $0.5 d^{k}-\epsilon g(d)$, respectively, where $g(d)=\bar{\lambda}^{0}+\sum_{h \in K \backslash k} d^{h} \bar{\lambda}^{h}$. These flows are obviously positive if 


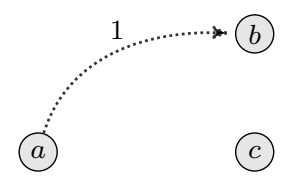

(a) $y^{k_{1} k_{1}}$

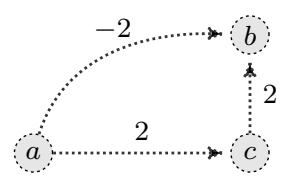

(b) $f^{0 k_{1}}$

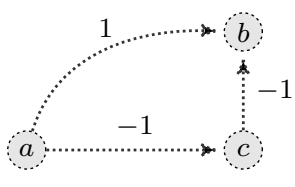

(c) $y^{k_{1} k_{2}}$

Figure 4: Non-static affine routing for commodity $k_{1}$.

either $g(d)=0$ or $\epsilon<d^{k} /|2 g(d)|$ for all $d \in \operatorname{vert}(\mathcal{D})$ with $g(d) \neq 0$. Such an $\epsilon$ exists since vert $(\mathcal{D})$ is finite.

Combining Proposition 9 with Theorem 10, we have completely described polytopes for which affine routings and static routings are equivalent, assuming that $G$ is acyclic. However, Proposition 9 is wrong for graphs containing cycles because $d \in \mathcal{D}_{0}^{k}$ does not imply that $f^{k}(d)=0$. It is possible that $\operatorname{dim}\left(\mathcal{D}_{0}^{k}\right)=|K|-1$ and that $f^{k}(d)$ describes a positive circulation. In Example 2 we saw that we can construct a graph $G$ for which it is possible to decompose a positive circulation into circulations that are not positive. Hence, one can check that using a properly defined uncertainty polytope $\mathcal{D}$ that fulfills the assumption of Proposition 9 it is possible to construct an irreducible affine routing for $G$ that is not static, which yields a counter-example to Proposition 9 for general graphs.

Proposition 8 and 9 state conditions on $\mathcal{D}$ for irreducible affine routings to be static which means that the corresponding solution costs coincide. However, it is possible that opt aff $=o p t_{\text {stat }}$ even if there exist irreducible affine routings that are not static.

Example 4. Consider the network design problem from Example 1 with the uncertainty set $\mathcal{D}$ defined by the extreme points $d_{1}=(2,1), d_{2}=(0,2)$, and $d_{3}=(1,1)$. The optimal solutions to this problem using static, affine, and dynamic routing have a cost of 9 . The corresponding capacity allocation is depicted in Figure 1(c), A feasible routing within these capacities is given by (statically) sending $50 \%$ of commodity $k_{1}$ along $a \rightarrow c \rightarrow b$ and $50 \%$ along $a \rightarrow b$ while $100 \%$ of commodity $k_{2}$ is sent along $a \rightarrow c$.

However, it can be easily seen that there exist affine routings that are not static within the same capacities. One such affine routing for commodity $k_{1}$ is depicted in Figure 4 keeping the static routing for $k_{2}$ (Figure $1(\mathrm{f})$.

Notice that the digraph $G$ is acyclic and that $\mathcal{D}$ does not fulfill the condition of Proposition 9

\subsection{Relation to dynamic routing}

Proposition 8 identifies demand polytopes for which affine routing is no better than static routing. However, we saw in Example 1 that affine routing may also perform as well as dynamic routing 
does, yielding strictly cheaper capacity allocations. For general robust optimization problems, Bertsimas and Goyal [16] show that affine recourse is equivalent to dynamic recourse when $\mathcal{D}$ is a simplex as for instance in Examples 1 and 4 Here we show that in the context of robust network design this condition is also necessary. In view of Lemma 2 , we can restrict our attention to routings serving the set of extreme points $\operatorname{vert}(\mathcal{D})$ in the following.

Proposition 11. All dynamic routings serving $\operatorname{vert}(\mathcal{D})$ are affine routings if and only if $\mathcal{D}$ is a simplex with dimension $m \leq|K|$.

Proof. Given a subset $S \subseteq \mathcal{D}$ of demand scenarios, we consider an arbitrary routing $f$ serving all $d \in S$. We further consider the linear equation system

$$
f_{a}^{0 k}+\sum_{h \in K} d^{h} y_{a}^{k h}=f_{a}^{k}(d), \quad d \in S
$$

for every $a \in A$ and $k \in K$, where the right hand sides correspond to the chosen routings $f(d)$ for $d \in S$.

If $\mathcal{D}$ is a simplex, then all its vertices are affinely independent, which implies that the left hand side rows of system $(23)$ with $S=\operatorname{vert}(\mathcal{D})$ are linearly independent. It follows that for $S=\operatorname{vert}(\mathcal{D})$ system (23) has a solution. Hence, by the definition of affine routings, every routing $f$ serving the vertices $\operatorname{vert}(\mathcal{D})$ of a simplex $\mathcal{D}$ is affine.

Let us assume $\mathcal{D}$ is not a simplex and let us first assume $\mathcal{D}$ is full-dimensional. Hence $\mathcal{D}$ has at least $|K|+2$ vertices. To construct a routing $f$ which is not affine we start with an affine routing for a subset $S$ of the extreme points. Let $S \subset \operatorname{vert}(\mathcal{D})$ be a subset of $|K|+1$ affinely independent vectors in $\operatorname{vert}(\mathcal{D})$. For every commodity $k \in K$ we take arbitrary flows $f^{k}(d), d \in S$, serving the individual vertices $S$ and for each $a \in A$, we solve the system 23 which has a unique solution $\left(\bar{f}_{a}^{0 k}, \bar{y}_{a}^{k}\right)$. This procedure yields an affine routing $f$ serving $S$ together with the defining multipliers $\left(\bar{f}^{0}, \bar{y}\right)$. Notice that any affine routing serving $S$ can be constructed this way. Now for $d \in \operatorname{vert}(\mathcal{D}) \backslash S$ we choose flows $f^{k}(d)$ serving $d$ such that

$$
f^{k}(d) \neq \bar{f}^{0 k}+\sum_{h \in K} d^{h} \bar{y}^{k h}
$$

This can always been done because there exist at least two paths between $s(k)$ and $t(k)$. These paths can be used to perturb any feasible flow by adding circulations. Hence, we have just constructed a routing serving all vertices $\operatorname{vert}(\mathcal{D})$ that is not affine.

If $\operatorname{dim}(\mathcal{D})=m<|K|, \mathcal{D}$ is included in an affine space defined by $|K|-m$ independent linear equations. We can use these equations to express $|K|-m$ components of the demand as affine functions of the others:

$$
d^{k}=\sum_{h \in Q} \alpha^{k h} d^{h}, \quad k \in K \backslash Q,
$$


where $|Q|=m$. Plugging (24) into the definition of an affine routing (8), any affine routing serving $\mathcal{D}$ can be expressed as an affine function of $m$ variables. Since $\mathcal{D}$ is not a simplex, it has at least $m+2$ extreme points. We choose $S$ as an arbitrary subset of $m+1$ affinely independent vectors in $\operatorname{vert}(\mathcal{D})$ and the rest of the proof is similar.

Proposition 11 does not imply that all dynamic routings serving a simplex $\mathcal{D}$ are affine. Indeed, any dynamic routing described by a non-linear function $f: \mathcal{D} \rightarrow \mathbb{R}_{+}^{|A| \times|K|}$ cannot be described by an affine routing. For instance, consider $\mathcal{D}=[0,1]$ and the dynamic routing that routes $d \in[0,0.5]$ along $p_{1}$ and $\left.\left.d \in\right] 0.5,1\right]$ along $p_{2}$. This routing is not affine as it is not described by a continuous function. However, there exists an affine routing (in the same capacities) that yields the same flow for each $d \in \operatorname{vert}(\mathcal{D})=\{0,1\}$. This affine routing is simply the static routing that routes any demand vector along $p_{2}$.

Example 5 shows that when the conditions of Propositions 8 and 11 are not fulfilled, that is, $\mathcal{D}$ is not a simplex and does not contain the origin, capacity allocation costs required by static, affine, and dynamic routings can be strictly different.

Example 5. Consider the network design problem from Example 1 with the uncertainty set $\mathcal{D}$ defined by the extreme points $d_{1}=(3,0), d_{2}=(0,3), d_{3}=(2,2)$, and $d_{4}=(0.5,0.5)$. The optimal capacity allocation costs with static, affine, and dynamic routings are, respectively, $13+\frac{1}{2}, 13+\frac{1}{3}$, and 13 . Notice that moving $d_{4}$ along the segment $(0,0)-(1,1)$ leaves static and dynamic optimal capacity allocations unchanged while the affine solution cost moves between 13 and $13+\frac{1}{2}$. In particular, if $d_{4}$ is set to $(0,0)$, the affine and static costs are the same, which we knew already from Proposition 8 . If $d_{4}$ is in $\operatorname{conv}\left\{d_{1}, d_{2}, d_{3},(1,1)\right\}$, the affine and dynamic costs are the same.

Propositions 8 and 11 relate theoretically affine routing to the well-known static and dynamic routings. Combining these results we obtain conditions on the demand set $\mathcal{D}$ which yield that dynamic routings are static and which establish that $o p t_{s t a t}=o p t_{d y n}$.

Corollary 12. Let the demand polytope $\mathcal{D}$ be the convex hull of $\{0\} \cup\left\{\epsilon_{h} e^{h}, h \in K\right\}$ with $\epsilon_{h}>0$ for all $h \in K$. Then, all dynamic routings serving $\operatorname{vert}(\mathcal{D})$ are static routings.

Note that there exist other situations in which dynamic routing is not better than static routing. The set $\mathcal{D}$ in Example 4 does not satisfy the hypothesis from Corollary 12 but $o p t_{s t a t}=o p t_{d y n}$. We refer to Frangioni et al. 29] for providing conditions on $\mathcal{D}$ for $o p t_{\text {stat }}=o p t_{\text {dyn }}$ to hold. Besides the structure of $\mathcal{D}$ these situations, however, also depend on the topology of $G$ and the design cost function $\kappa$. 


\section{Computational experiments}

In this section we investigate the objective gaps between optimal networks designs using static, affine, and dynamic routings, respectively, facing network topologies from the SNDlib [43]. We start by defining the demand uncertainty polytope $\mathcal{D}$ our computations rely on.

\subsection{Uncertainty sets and data}

Two polyhedral uncertainty sets and their variations receive most of the attention among robust network design practitioners, the hose model going back to Duffield et al. [26] and defined in Example 3, see also [2, 10, 38, and a restricted interval uncertainty set inspired by the work of Bertsimas and Sim [17, 18. We refer to this latter model as the $\Gamma$-model, see for instance [2, 35, 36, 45]. Both models are combined in Altin et al. 3].

One of the key advantages of the hose model is the fact that it does not require any estimates of pairwise demands. The potential demands are described by upper bounds on the traffic at the source and target nodes. However, as there are no implied lower bounds we conclude in Example 3 that $o p t_{\text {stat }}=o p t_{\text {aff }}$ for the hose model based on Proposition 8. We can in general not expect any improvements on the network cost for the hose model when using affine routing unless we explicitly impose lower bounds on the traffic as in Altin et al. [3].

In the $\Gamma$-model, we assume that the traffic $d^{k}$ for commodity $k$ varies around a mean-demand $\bar{d}^{k}>0$ with a maximal possible deviation of $0 \leq \hat{d}^{k} \leq \bar{d}^{k}$; that is,

$$
d^{k} \in\left[\bar{d}^{k}-\hat{d}^{k}, \bar{d}^{k}+\hat{d}^{k}\right] \text { for all } k \in K .
$$

The $\Gamma$-model now limits the number of simultaneous deviations from the mean by the value $\Gamma \in\{0, \ldots,|K|\}$; that is,

$$
\sum_{k \in K}\left|d^{k}-\bar{d}^{k}\right| / \hat{d}^{k} \leq \Gamma
$$

Of course $d^{k}>0$ as long as $\bar{d}^{k}>\hat{d}^{k}$. Hence the conditions of Proposition 8 do not apply in general for the $\Gamma$-model (affine routing might improve over static routing).

The corresponding uncertainty polytope $\mathcal{D}$ can be described in $\mathbb{R}^{|K|}$ directly using exponentially many linear inequalities or alternatively using a compact extended formulation. For the latter, we rewrite $d^{k}=\bar{d}^{k}+\left(\sigma_{+}^{k}-\sigma_{-}^{k}\right) \hat{d}^{k}$ for all $k \in K$ and let $\left(\sigma_{+}, \sigma_{-}\right) \in \mathcal{D}^{\sigma}$, where

$$
\mathcal{D}^{\sigma}:=\left\{\left(\sigma_{+}, \sigma_{-}\right) \in \mathbb{R}_{+}^{2|K|}: \sum_{k \in K}\left(\sigma_{+}^{k}+\sigma_{-}^{k}\right) \leq \Gamma \text { and } \sigma_{+}^{k}+\sigma_{-}^{k} \leq 1 \text { for all } k \in K\right\} .
$$

The set $\mathcal{D}^{\sigma}$ corresponds to all possible deviation scenarios from the nominal vector $\bar{d}$. The number of simultaneous deviations is restricted independent of being downward or upward deviations.

For the static and the dynamic routing principle, by exploiting domination, respectively total domination (see Section 2 and [42]), it is possible to remove all vertices from $\mathcal{D}^{\sigma}$ that correspond 
to downward deviations and to consider uncertainty in the space $\sigma_{+}$only [2, 35, 36]. In this case $d^{k}=\bar{d}^{k}+\sigma_{+}^{k} \hat{d}^{k}$ for all $k \in K$ and we consider uncertain upward deviations $\sigma_{+} \in \mathcal{D}_{+}^{\sigma}$, where

$$
\mathcal{D}_{+}^{\sigma}:=\left\{\sigma_{+} \in \mathbb{R}_{+}^{|K|}: \sum_{k \in K} \sigma_{+}^{k} \leq \Gamma \text { and } \sigma_{+}^{k} \leq 1 \text { for all } k \in K\right\} .
$$

Of course $\mathcal{D}_{+}^{\sigma}$ is a projection of $\mathcal{D}^{\sigma}$. It follows that for the static and dynamic routing principle it is equivalent to optimize using $\mathcal{D}^{\sigma}$ or $\mathcal{D}_{+}^{\sigma}$. Static as well as dynamic solutions supporting $\mathcal{D}_{+}^{\sigma}$ will automatically support $\mathcal{D}^{\sigma}$. Moreover, static and dynamic routings might even cover demandscenarios outside the actual deviation polytope $\mathcal{D}^{\sigma}$ as long as these are (totally) dominated, e.g., demand vectors with $d^{k}=\epsilon$ for all $k \in K$ and $\epsilon>0$ small enough. By Proposition 6, this is not true for the affine routing principle. It can not be said a priori whether or not affine solutions supporting $\mathcal{D}_{+}^{\sigma}$ will support deviations in $\mathcal{D}^{\sigma}$. All possible downward variations have to be included in the uncertainty definition since the feasibility of an affine routing can only be ensured if demand-deviations never leave the given uncertainty set. In the sequel we will use the set $\mathcal{D}_{+}^{\sigma}$ for static and dynamic scenarios. But in the case of affine recourse we will study optimal solutions for both the uncertainty set $\mathcal{D}_{+}^{\sigma}$ and $\mathcal{D}^{\sigma}$. Notice that for $\Gamma>0$ both $\mathcal{D}_{+}^{\sigma}$ and $\mathcal{D}^{\sigma}$ are full-dimensional since they contain $\epsilon_{k} e^{k}$ with $\epsilon_{k}>0$ for each $k \in K$ and the origin.

Remark 1. A simple (also compact) alternative to the $\Gamma$-model is to use 25 plus a relaxation of $(26)$ :

$$
\sum_{k \in K} \frac{d^{k}-\bar{d}^{k}}{\hat{d}^{k}} \leq \Gamma
$$

In this case there might be more than $\Gamma$ many upward deviations if compensated by an appropriate number of downward deviations and vice versa. Obviously, this results in a relaxed version of the model described above, potentially giving more conservative solutions.

We selected three instances janos-us, sun, and giul39 from SNDlib [43] which are feasible for a directed formulation as considered in this paper. These networks have 26/27/39 nodes and 84/102/172 arcs, respectively. More characteristics and statistics can be found on the SNDlib website [43. All instances contain a single demand scenario with directed node-to-node demand values. To parametrize the $\Gamma$-model we used these values as nominal demands $\bar{d}^{k}$ and fixed the maximum deviation to $\hat{d}^{k}:=0.4 \bar{d}^{k}$ for every commodity. That is, we made the assumption that the demand deviates by at most $40 \%$ from the nominal value. To reduce the size of the formulations and to be able to do a series of runs we considered the largest 10 to 50 commodities $k$ with respect to the value $\bar{d}^{k}$; that is, $|K|$ takes values in $\{10,20,30,50\}$. We considered all values $\Gamma$ in $\{1, \ldots, 7\}$. Notice that small values of $\Gamma$ are reasonable as they already introduce a relatively high level of robustness; see for instance [35, 36] and also check the values in Table 2 . 


\subsection{Robust counterparts}

The demand uncertainty polytope $\mathcal{D}^{\sigma}$ described by (27) has $2|K|$ variables and $|K|+1$ constraints (not counting non-negativity constraints). For $\mathcal{D}_{+}^{\sigma}$ the number of variables reduces to $|K|$. Consequently, the corresponding static and affine robust counterparts are compact, as shown in [5] (static routing) and in Section 3.1 (affine routing).

For the static case, a compact reformulation using the $\Gamma$-model can be found for instance in [35, 36. It includes flow template conditions 12 and a dualization of the capacity constraints which results in $2|A||K|+2|A|$ variables and $|K|(|V|-1)+(|K|+1)|A|$ constraints. Notice that always one flow conservation constraint per commodity can be omitted.

To set up the affine robust counterpart we dualized the capacity constraints and the flow non-negativity constraints following model $(A A R N D)$ described in Section 3 Notice that $\mathcal{D}^{\sigma}$ is described in dimension $2|K|$ which increases the number of rows of the dualization and hence the number of rows of the model $(A A R N D)$. The resulting affinely adjustable robust network design formulation $(A A R N D)$ for the $\Gamma$-model with uncertainty set $\mathcal{D}^{\sigma}$ has $2|A||K|^{2}+3|A||K|+2|A|$ variables and $\left(|K|^{2}+|K|\right)(|V|-1)+2|A||K|^{2}+3|A||K|+|A|$ constraints. If the set $\mathcal{D}_{+}^{\sigma}$ is used instead, the number of constraints reduces to $\left(|K|^{2}+|K|\right)(|V|-1)+|A||K|^{2}+2|A||K|+|A|$. Again we can omit one flow conservation constraint per commodity in the routing template 12 . Also one flow conservation constraint per commodity of the flow cycle conditions (13) and (14) is redundant.

To calculate dynamic optimal solutions we used $(R N D)$ introduced in Section 2 restricted to those $d \in \mathcal{D}$ that correspond to the vertices of the demand uncertainty polytope. As explained above it suffices to consider non-dominated vertices corresponding to extreme upward deviations scenarios. These are the demand-vectors where $\Gamma$ out of $|K|$ commodities are at their peak values $\bar{d}^{k}+\hat{d}^{k}$ and the remaining $|K|-\Gamma$ commodities are at their nominal values $\bar{d}^{k}$. More precisely, in terms of deviation scenarios, for every subset $Q \subseteq K$ with $|Q|=\Gamma$ we have to consider the vertex $\sigma_{+} \in \mathcal{D}_{+}^{\sigma}$ with $\sigma_{+}^{k}=1$ for $k \in Q$ and $\sigma_{+}^{k}=0$ for $k \in K \backslash Q$, which results in $\left(\begin{array}{c}|K| \\ \Gamma\end{array}\right)$ many vertices (totally dominating all other vertices of $\mathcal{D}_{+}^{\sigma}$ ). Consequently, the resulting exponential model to solve the dynamic robust network design problem has $\left(\begin{array}{c}|K| \\ \Gamma\end{array}\right)|A||K|+|A|$ variables and $\left(\begin{array}{c}|K| \\ \Gamma\end{array}\right)(|K|(|V|-1)+|A|)$ constraints. Notice that we can sensibly reduce the problem size for the dynamic case, by aggregating commodities with a common source (or a common destination) node [19, 37. For our instances $|K|$ could be reduced from 50 to 18 (resp. 10 and 6) for janos-us (resp. giul39 and sun). It is however easy to show that such aggregation is not compatible with static and affine routing principles.

For comparison purposes we summarize the sizes of the three different models in Table 1.

Remark 2. As we can conclude from Table 1 affine and dynamic models can be very large even 


\begin{tabular}{lrr}
\hline model & rows & columns \\
\hline static $\mathcal{D}^{\sigma}$ & $|K|(|V|-1)+(|K|+1)|A|$ & $2|A||K|+2|A|$ \\
affine $\mathcal{D}^{\sigma}$ & $\left(|K|^{2}+|K|\right)(|V|-1)+2|A||K|^{2}+3|A||K|+|A|$ & $2|A||K|^{2}+3|A||K|+2|A|$ \\
affine $\mathcal{D}_{+}^{\sigma}$ & $\left(|K|^{2}+|K|\right)(|V|-1)+|A||K|^{2}+2|A||K|+|A|$ & $2|A||K|^{2}+3|A||K|+2|A|$ \\
& $\left(\begin{array}{c}|K| \\
\Gamma\end{array}\right)(|K|(|V|-1)+|A|)$ & $\left(\begin{array}{c}|K| \\
\Gamma\end{array}\right)|A||K|+|A|$ \\
\hline
\end{tabular}

Table 1: Model sizes with respect to the number of nodes $|V|$, arcs $|A|$, commodities $|K|$, and the value $\Gamma$.

for medium sized instances. In general it might be wise to use decomposition methods to solve the corresponding problems which is beyond the scope of this paper. Very few publications in robust network design (beyond static routing) consider algorithmic issues or present solutions. Implementing well-engineered solution algorithms for $(R N D)$ with affine respectively dynamic routing remains a challenging task. However, a few steps in this direction have been carried out.

Ouorou and Vial [45] use a path-flow model and a column generation based approach to solve the affine robust counterpart. Babonneau et al. 7] solve a similar problem after restricting the set of admissible paths and also restricting the number of affine decision variables. For the intractable design problem with integral capacities and dynamic routing, Mattia [38] shows that small to medium sized instances can be solved in a reasonable amount of time when using a branchand-cut approach based on Benders decomposition [15]. To solve the coNP-complete separation problem for robust metric inequalities [33, 40], Mattia [38] uses mixed integer programs with big-M coefficients.

\section{$4.3 \quad$ Results}

Our numerical results are summarized in Table 2 and Table 3 . For our computations we used the interior point (barrier) solver of CPLEx 12.1 [32] on a 64bit 3.0 GHz Quad-Core CPU with 8GB of memory allowing for 4 threads and 8 hours of CPU time for every individual run. Since we are only interested in the objective value of the optimal solution we switched off the crossover of Cplex. LP models have been set up using the modeling language ZimPL [34].

The first three columns of Table 2 state the instance name followed by the number of commodities $|K|$ and the size of $\Gamma$. The value $\Phi$ in column 4 indicates the largest number of commodities using the same arc in the optimal static solution as discussed below. We comment on $\Phi$ in the lemma further on. Column 5 gives the static optimal objective value $\operatorname{opt}_{\text {stat }}(K, \Gamma)$. The last three columns state the percentage affine gap $100\left(1-\operatorname{opt}_{a f f}(K, \Gamma) / o p t_{\text {stat }}(K, \Gamma)\right)$, where 
$\operatorname{opt}_{\text {aff }}(K, \Gamma)$ corresponds to the optimal solution using the uncertainty set $\mathcal{D}^{\sigma}$, the percentage upward affine gap $100\left(1-o p t_{a f f}^{+}(K, \Gamma) / o p t_{\text {stat }}(K, \Gamma)\right)$ using $\mathcal{D}_{+}^{\sigma}$, and the percentage dynamic gap 100(1-opt dyn $\left.(K, \Gamma) / o p t_{\text {stat }}(K, \Gamma)\right)$, respectively. Letters $\mathrm{T}$ and $\mathrm{M}$ indicate that the time or memory limit has been reached, respectively. Table 2 clearly shows the relation

$$
o p t_{d y n}(K, \Gamma) \leq o p t_{a f f}^{+}(K, \Gamma) \leq o p t_{a f f}(K, \Gamma) \leq o p t_{\text {stat }}(K, \Gamma)
$$

In general we observe that the dynamic gap is relatively small. It is below $11 \%$ for all solved scenarios. It seems that for practical networks with a modest number of demands the cost of static solutions is fairly close to the optimal (dynamic) design cost. In particular for small $|K|$ and larger $\Gamma$ the dynamic gap is extremely small and is even $0 \%$ in many cases. Notice that it always holds that $\operatorname{opt}_{\text {stat }}(K,|K|)=\operatorname{opt}_{\text {dyn }}(K,|K|)$ since for $\Gamma=|K|$ there is only one non-dominated vertex of the uncertainty polytope $\mathcal{D}^{\sigma}$. In this case we solve the nominal problem for the single worst-case demand-matrix having all demands at their peak. However, it can also be clearly seen that the dynamic gap increases with the number of considered demands. Notice that Mattia 38, shows that also for larger commodity sets the dynamic gap is extremely small if discrete capacities are considered. In contrast, Ouorou [44] observes that the affine gap can be significantly larger, about $30 \%$, when one introduces limitations on the number of different paths that can be used to route every commodity. Such restrictions are common for instance in the design and engineering of telecommunications networks.

Even more interesting, Table 2 indicates that all affine solutions are almost optimal, i.e., the corresponding cost is very close to the dynamic cost. In particular the upward affine gap (considering $\mathcal{D}_{+}^{\sigma}$ ) in many cases even coincides with the dynamic gap, when available. For $\Gamma=1$ the uncertainty set $\mathcal{D}_{+}^{\sigma}$ is a simplex with $|K|+1$ vertices, hence $o p t_{a f f}^{+}(K, 1)=o p t_{d y n}(K, 1)$ by Proposition 11 But also for $\Gamma>1$ it often holds that $o p t_{a f f}^{+}(K, \Gamma) \approx \operatorname{opt}_{d y n}(K, \Gamma)$. As mentioned above the corresponding affine routing does not necessarily support demands in $\mathcal{D}^{\sigma} \backslash \mathcal{D}_{+}^{\sigma}$. But even considering downward deviations by using the uncertainty set $\mathcal{D}^{\sigma}$ for affine recourse does not remarkably decrease the corresponding gap. Affine solutions for $\mathcal{D}^{\sigma}$ are still very close to the dynamic solutions and clearly improve on the static solutions in terms of capacity cost.

\begin{tabular}{rrrrrrrr} 
instance $|K|$ & $\Gamma$ & $\Phi$ & $\begin{array}{r}\text { static } \\
\text { opt }_{\text {stat }}\end{array}$ & $\begin{array}{r}\text { affine } \mathcal{D}^{\sigma} \\
\text { gap in } \%\end{array}$ & $\begin{array}{r}\text { affine } \mathcal{D}_{+}^{\sigma} \\
\text { gap in } \%\end{array}$ & $\begin{array}{r}\text { dynamic } \\
\text { gap in \% }\end{array}$ \\
\hline 10 & 1 & 2 & $3.149202 \mathrm{e}+05$ & 4.9 & 5.7 & 5.7 \\
10 & 2 & 1 & $3.323827 \mathrm{e}+05$ & 0.0 & 0.0 & 0.0 \\
20 & 1 & & 3 & $4.657367 \mathrm{e}+05$ & 6.4 & 7.2 & 7.2 \\
20 & 2 & 2 & $5.125317 \mathrm{e}+05$ & 5.1 & 6.2 & 6.5 \\
20 & 3 & 2 & $5.125317 \mathrm{e}+05$ & 0.1 & 0.9 & 2.5 \\
janos-us & 20 & 4 & 2 & $5.125317 \mathrm{e}+05$ & 0.0 & 0.0 & 0.0
\end{tabular}




\begin{tabular}{|c|c|c|c|c|c|}
\hline 20 & 5 & $25.125317 \mathrm{e}+05$ & 0.0 & 0.0 & M \\
\hline 30 & 1 & $4 \quad 6.127240 \mathrm{e}+05$ & 6.8 & 7.5 & 7.5 \\
\hline 30 & 2 & $5 \quad 6.722822 \mathrm{e}+05$ & 7.5 & 8.3 & 8.7 \\
\hline 30 & 3 & $5 \quad 6.988964 \mathrm{e}+05$ & 5.7 & 6.6 & 7.0 \\
\hline 30 & 4 & $36.992080 \mathrm{e}+05$ & 1.4 & 2.4 & M \\
\hline 30 & 5 & $36.992080 \mathrm{e}+05$ & 0.0 & 0.0 & M \\
\hline 40 & 1 & $56.729093 \mathrm{e}+05$ & 7.5 & 8.2 & 8.2 \\
\hline 40 & 2 & $57.324675 \mathrm{e}+05$ & 7.5 & 8.4 & 8.8 \\
\hline 40 & 3 & $5 \quad 7.631223 \mathrm{e}+05$ & 5.6 & 6.7 & M \\
\hline 40 & 4 & $47.659107 \mathrm{e}+05$ & 1.9 & 2.9 & M \\
\hline 40 & 5 & $47.659107 \mathrm{e}+05$ & 0.0 & 0.2 & M \\
\hline 40 & 6 & $47.659107 \mathrm{e}+05$ & 0.0 & 0.0 & M \\
\hline 50 & 1 & $5 \quad 7.311094 \mathrm{e}+05$ & 7.7 & 8.4 & 8.4 \\
\hline 50 & 2 & $5 \quad 7.925296 \mathrm{e}+05$ & 7.8 & 8.7 & 9.1 \\
\hline 50 & 3 & $58.266402 \mathrm{e}+05$ & 6.3 & 7.4 & M \\
\hline 50 & 4 & $58.369683 \mathrm{e}+05$ & 3.7 & 4.8 & M \\
\hline 50 & 5 & $48.386076 \mathrm{e}+05$ & 1.1 & 2.1 & M \\
\hline 50 & 6 & $48.386076 \mathrm{e}+05$ & 0.0 & 0.0 & M \\
\hline 10 & 1 & $32.616416 \mathrm{e}+02$ & 7.5 & 9.8 & 9.8 \\
\hline 10 & 2 & $32.740441 \mathrm{e}+02$ & 0.6 & 2.7 & 3.0 \\
\hline 10 & 3 & $22.753181 \mathrm{e}+02$ & 0.0 & 0.0 & 0.4 \\
\hline 10 & 4 & $22.753181 \mathrm{e}+02$ & 0.0 & 0.0 & 0.0 \\
\hline 10 & 5 & $22.753181 \mathrm{e}+02$ & 0.0 & 0.0 & 0.0 \\
\hline 10 & 6 & $22.753181 \mathrm{e}+02$ & 0.0 & 0.0 & 0.0 \\
\hline 10 & 7 & $22.753181 \mathrm{e}+02$ & 0.0 & 0.0 & 0.0 \\
\hline 20 & 1 & $5 \quad 4.314919 \mathrm{e}+02$ & 8.1 & 9.9 & 9.9 \\
\hline 20 & 2 & $5 \quad 4.666696 \mathrm{e}+02$ & 6.1 & 8.9 & 9.2 \\
\hline 20 & 3 & $5 \quad 4.821624 \mathrm{e}+02$ & 3.2 & 5.5 & 6.4 \\
\hline 20 & 4 & $5 \quad 4.867587 \mathrm{e}+02$ & 0.8 & 2.0 & 3.5 \\
\hline 20 & 5 & $4 \quad 4.878762 \mathrm{e}+02$ & 0.3 & 0.7 & M \\
\hline 20 & 6 & $4 \quad 4.878762 \mathrm{e}+02$ & 0.0 & 0.2 & M \\
\hline 20 & 7 & $4 \quad 4.878762 \mathrm{e}+02$ & 0.0 & 0.0 & M \\
\hline 30 & 1 & $75.563141 \mathrm{e}+02$ & 8.0 & 9.2 & 9.2 \\
\hline 30 & 2 & $7 \quad 6.029896 \mathrm{e}+02$ & 8.1 & 10.1 & 10.5 \\
\hline 30 & 3 & $86.303494 \mathrm{e}+02$ & 6.6 & 8.8 & 9.6 \\
\hline 30 & 4 & $7 \quad 6.400667 \mathrm{e}+02$ & 3.6 & 5.6 & M \\
\hline 30 & 5 & $7 \quad 6.465764 \mathrm{e}+02$ & 1.8 & 3.1 & M \\
\hline 30 & 6 & $7 \quad 6.491593 \mathrm{e}+02$ & 0.9 & 1.8 & M \\
\hline 30 & 7 & $66.500533 \mathrm{e}+02$ & 0.4 & 0.8 & $\mathrm{M}$ \\
\hline
\end{tabular}




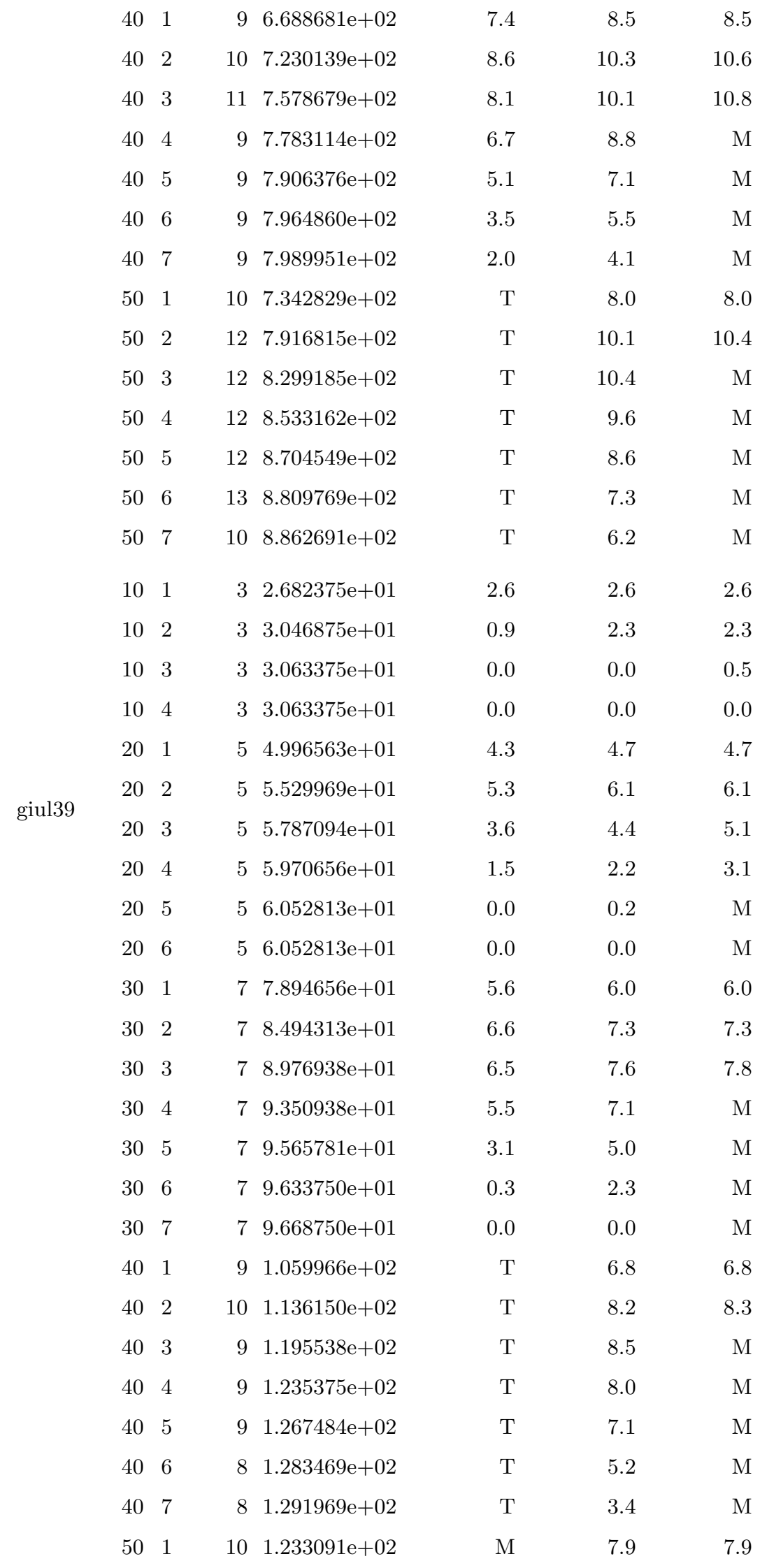




\begin{tabular}{rrrrccr}
50 & 2 & 10 & $1.325416 \mathrm{e}+02$ & $\mathrm{M}$ & 9.8 & 10.2 \\
50 & 3 & 10 & $1.385434 \mathrm{e}+02$ & $\mathrm{M}$ & 9.7 & $\mathrm{M}$ \\
50 & 4 & 10 & $1.432656 \mathrm{e}+02$ & $\mathrm{M}$ & 9.7 & $\mathrm{M}$ \\
50 & 5 & 10 & $1.468328 \mathrm{e}+02$ & $\mathrm{M}$ & 9.0 & $\mathrm{M}$ \\
50 & 6 & 9 & $1.493625 \mathrm{e}+02$ & $\mathrm{M}$ & 7.8 & $\mathrm{M}$ \\
50 & 7 & 9 & $1.502844 \mathrm{e}+02$ & $\mathrm{M}$ & 5.8 & $\mathrm{M}$ \\
\hline
\end{tabular}

Table 2: Comparing static, affine, and dynamic routing in terms of optimality gap. $\Gamma$-model with $\Gamma \in\{1, \ldots, 7\}$ and $|K| \in\{10,20,30,40,50\}$. We removed rows whenever objective values and gaps did not differ from the previous row.

Clearly, we could not solve all affine and in particular not all dynamic instances. However, the results above at least indicate that the affine routing principle allows enough flexibility to almost capture dynamic routings. It also suggests the following general approach. Given a general uncertainty polytope $\mathcal{D}$, in order to calculate a cheap network together with a feasible dynamic routing, one may compute the cost-minimal affine solution (capacity and routing) for $\mathcal{D}$ instead. For an even cheaper still (dynamically) feasible capacity allocation one might remove all vertices from $\mathcal{D}$ that are (totally) dominated and then compute the cost-minimal affine solution. In the latter case the resulting affine routing is not necessarily feasible for $\mathcal{D}$ but there exists a dynamic routing for the computed capacity allocation, and this capacity allocation can be considered as being almost optimal. In fact, if only the capacity allocation and its cost are of interest, affine recourse with such a reduced uncertainty set can be used to approximate the cost for free recourse. Notice that in our case, using the $\Gamma$-model, the affine robust counterpart $(A A R N D)$ for the reduced set $\mathcal{D}_{+}^{\sigma}$ was much easier to solve than $(A A R N D)$ for the original set $\mathcal{D}^{\sigma}$; see Table 3 .

In the following we will prove special properties of the $\Gamma$-model which can be observed in Table2 For this we have to introduce some notation. We define the numbers $\Phi(K, \Gamma), \Gamma^{\star}(K) \in \mathbb{Z}_{+}$ which depend on the structure of the network and commodities. For a scenario pair $(K, \Gamma)$ and an optimal static solution $S=(x, y)$ with objective value $\operatorname{opt}_{\text {stat }}(K, \Gamma)$, let $\Phi(K, \Gamma, S)$ denote the largest number of commodities using the same arc in the solution $S$; that is,

$$
\Phi(K, \Gamma, S):=\max \left\{\left|\left\{k \in K: y_{a}^{k}>0\right\}\right|: a \in A\right\} .
$$

We set

$$
\Phi(K, \Gamma):=\min \{\Phi(K, \Gamma, S): S \text { static optimal }\}
$$

that is, $\Phi(K, \Gamma)$ gives the smallest value $\Phi(K, \Gamma, S)$ among all optimal static solutions $S$. With this definition the value $\Phi$ in column 5 of Table 2 is an upper bound on $\Phi(K, \Gamma)$ since it states 
the value $\Phi(K, \Gamma, S)$ for one of the possible optimal static solutions $S$. For commodity set $K$ we define

$$
\Gamma^{\star}(K):=\min \left\{\Gamma: \Gamma \in \mathbb{Z}_{+}, \Gamma \geq \Phi(K, \Gamma)\right\} .
$$

In any solution $S$ at most $|K|$ commodities can meet, hence $\Phi(K, \Gamma) \leq \Phi(K, \Gamma, S) \leq|K|$. We also conclude that $\Phi(K, \Gamma) \geq 1$ since in all solutions at least one arc is used (as long as $K$ is not empty). It follows directly that $1 \leq \Gamma^{\star}(K) \leq|K|$.

In the following we essentially show that for fixed $K$ and increasing $\Gamma$ at some point the optimal solution value opt $_{\text {stat }}(K, \Gamma)$ remains constant and is based on a shortest path solution; that is, an optimal static solution can be obtained by routing all commodities on a shortest path with respect to the arc costs $\kappa_{a}, a \in A$. The corresponding threshold for $\Gamma$ is precisely $\Gamma^{\star}(K)$.

Lemma 13. Let $G=(V, A), K \neq \emptyset$, and $\Gamma \geq \Gamma^{\star}(K)$. Then

- $o p t_{\text {stat }}(K, \Gamma)=o p t_{\text {stat }}\left(K, \Gamma^{\star}(K)\right)$,

- $\Phi(K, \Gamma)=\Phi\left(K, \Gamma^{\star}(K)\right) \leq \Gamma$, and

- scenario $(K, \Gamma)$ has an optimal static solution with a shortest path template.

Proof. Let $S$ be an optimal static solution for scenario $\left(K, \Gamma^{\star}(K)\right)$ such that $\Phi\left(K, \Gamma^{\star}(K), S\right)=$ $\Phi\left(K, \Gamma^{\star}(K)\right)$. There are at most $\Phi\left(K, \Gamma^{\star}(K)\right) \leq \Gamma^{\star}(K)$ commodities meeting on every individual arc in this solution. Hence, for every arc, all the commodities can be at their peak simultaneously. It follows that solution $S$ is also optimal for any scenario $(K, \Gamma)$ with $\Gamma \geq \Gamma^{\star}(K)$ with the same objective; that is, opt stat $(K, \Gamma)=o p t_{\text {stat }}\left(K, \Gamma^{\star}(K)\right)$ for all $\Gamma \geq \Gamma^{\star}(K)$. To see this notice that for arbitrary scenarios and solutions the solution value cannot decrease if we increase $\Gamma$. For solution $S$ and scenarios $(K, \Gamma)$ with $\Gamma \geq \Gamma^{\star}(K)$ the solution value can also not increase as we have to consider all commodities at their peak anyway. Also $\Phi(K, \Gamma)=\Phi\left(K, \Gamma^{\star}(K)\right)$ for all $\Gamma \geq \Gamma^{\star}(K)$. Otherwise $\Phi\left(K, \Gamma^{\star}(K), S\right)$ was not minimal for scenario $\left(K, \Gamma^{\star}(K)\right)$.

To prove the third claim observe that $\operatorname{opt}_{\text {stat }}(K,|K|)=o p t_{\text {stat }}\left(K, \Gamma^{\star}(K)\right)$. But every optimal static solution $S^{\prime}=(x, y)$ to scenario $(K,|K|)$ is based on a shortest path template $y$. These shortest path solutions are obviously optimal for any scenario $(K, \Gamma)$ with $\Gamma \geq \Gamma^{\star}(K)$.

Note that as a consequence of Lemma 13 there always exists a shortest path template for set $K$ where at most $\Phi\left(K, \Gamma^{\star}(K)\right)$ commodities meet on every arc.

Comparing the value $\Gamma$ and the value $\Phi$ in columns 3 and 4 of Table 2 we obtain $\Gamma^{\star}(K)=$ 2, 2, 4, 4, 5 for network janos-us and $|K|=10,20,30,40,50$, respectively. For network sun and giul39 we have $\Gamma^{\star}(K)=3,5,7$ for $|K|=10,20,30$, respectively. For all networks the values $\Gamma^{\star}(K)$ are very small but increasing with the number of commodities. Notice that since $o p t_{\text {stat }}\left(K, \Gamma^{\star}(K)\right)=$ opt $_{\text {stat }}(K,|K|)$ the worst-case objective for the $\Gamma$-model (together with a 
shortest path solution) is obtained already early (for small $\Gamma$ ) with static recourse. Affine and dynamic solutions tend to admit the worst case later (for larger $\Gamma$ ).

Table 3 reports on the CPU time used to the solve the models. The first three columns of Table 3 again indicate the instance followed by the number of commodities $|K|$ and the size of $\Gamma$. Additionally, there are columns for every considered routing principle stating the number of non-zeroes (nonz) in the linear programming model and the CPU time in seconds (time). Since we were only interested in objective values we tried to solve all problems using the barrier method without crossover. It can be seen that all static models can be solved within 1 second of CPU time. The number of non-zeroes for the static and affine models is independent of the value $\Gamma$ and increases polynomially with the number of considered commodities. The affine models however are very large already for small values of $|K|$ with a huge number of non-zeroes (of the order of $10^{6}$ already for $\left.|K|=30,40,50\right)$. We could still solve all affine counterparts corresponding to $\mathcal{D}_{+}^{\sigma}$ in less than one hour for janos-us and sun and in less than two hours for giul39. In contrast, time and memory limits are reached when solving $(A A R N D)$ for $\mathcal{D}^{\sigma}$ when $|K| \geq 40$ (giul39) and $|K| \geq 50$ (sun). As expected the affine robust counterpart for the reduced set $\mathcal{D}_{+}^{\sigma}$ is much easier to solve because of a smaller LP; also see Table 1 .

As long as the number of non-zeroes is modest the dynamic models seem to be easier to solve than their affine counterparts. However, the number of non-zeroes is exponential both in the number of commodities and in the size of $\Gamma$. It exceeds $10^{9}, 10^{10}, 10^{11}$ for $\Gamma=7$ and $|K|=30,40,50$, respectively, so we can provide dynamic solutions only for very small values of $\Gamma$ or small values of $|K|$. In all other cases the memory limit was reached either already when setting up the LPs using ZIMPL or later in the barrier algorithm.

\begin{tabular}{|c|c|c|c|c|c|c|c|c|c|c|}
\hline \multirow[b]{2}{*}{ instance } & \multirow[b]{2}{*}{$|K|$} & \multirow[b]{2}{*}{$\Gamma$} & \multicolumn{2}{|c|}{ static } & \multicolumn{2}{|c|}{ affine $\mathcal{D}^{\sigma}$} & \multicolumn{2}{|c|}{ affine $\mathcal{D}_{+}^{\sigma}$} & \multicolumn{2}{|l|}{ dynamic } \\
\hline & & & nonz & time & nonz & time & nonz & time & nonz & time \\
\hline \multirow{13}{*}{ janos-us } & 10 & 1 & 5978 & 1 & 116998 & 27 & 81718 & 5 & 15540 & 1 \\
\hline & 10 & 2 & 5978 & 1 & 116998 & 15 & 81718 & 5 & 69930 & 1 \\
\hline & 10 & 3 & 5978 & 1 & 116998 & 16 & 81718 & 2 & 186480 & 1 \\
\hline & 10 & 4 & 5978 & 1 & 116998 & 4 & 81718 & 4 & 326340 & 2 \\
\hline & 10 & 5 & 5978 & 1 & 116998 & 3 & 81718 & 3 & 391608 & 2 \\
\hline & 10 & 6 & 5978 & 1 & 116998 & 3 & 81718 & 5 & 326340 & 2 \\
\hline & 10 & 7 & 5978 & 1 & 116998 & 2 & 81718 & 12 & 186480 & 1 \\
\hline & 20 & 1 & 11786 & 1 & 450786 & 240 & 313026 & 32 & 45800 & 1 \\
\hline & 20 & 2 & 11786 & 1 & 450786 & 336 & 313026 & 153 & 435100 & 4 \\
\hline & 20 & 3 & 11786 & 1 & 450786 & 279 & 313026 & 79 & 2610600 & 38 \\
\hline & 20 & 4 & 11786 & 1 & 450786 & 238 & 313026 & 22 & 11095050 & 111 \\
\hline & 20 & 5 & 11786 & 1 & 450786 & 248 & 313026 & 163 & 35504160 & M \\
\hline & 20 & 6 & 11786 & 1 & 450786 & 247 & 313026 & 180 & 88760400 & M \\
\hline
\end{tabular}




\begin{tabular}{|c|c|c|c|c|c|c|c|c|c|c|}
\hline & 20 & 7 & 11786 & 1 & 450786 & 261 & 313026 & 192 & 177520800 & M \\
\hline & 30 & 1 & 17594 & 1 & 1001534 & 1389 & 694094 & 88 & 76140 & 3 \\
\hline & 30 & 2 & 17594 & 1 & 1001534 & 1847 & 694094 & 153 & 1104030 & 13 \\
\hline & 30 & 3 & 17594 & 1 & 1001534 & 2161 & 694094 & 191 & 10304280 & 213 \\
\hline & 30 & 4 & 17594 & 1 & 1001534 & 2490 & 694094 & 255 & 69553890 & M \\
\hline & 30 & 5 & 17594 & 1 & 1001534 & 1802 & 694094 & 135 & 361680228 & M \\
\hline & 30 & 6 & 17594 & 1 & 1001534 & 1693 & 694094 & 157 & 1507000950 & M \\
\hline & 30 & 7 & 17594 & 1 & 1001534 & 1557 & 694094 & 141 & 5166860400 & M \\
\hline & 40 & 1 & 23410 & 1 & 1769570 & 3021 & 1225250 & 265 & 150640 & 7 \\
\hline & 40 & 2 & 23410 & 1 & 1769570 & 5362 & 1225250 & 407 & 2937480 & 49 \\
\hline & 40 & 3 & 23410 & 1 & 1769570 & 7823 & 1225250 & 672 & 37208080 & M \\
\hline & 40 & 4 & 23410 & 1 & 1769570 & 13192 & 1225250 & 802 & 344174740 & M \\
\hline & 40 & 5 & 23410 & 1 & 1769570 & 6657 & 1225250 & 672 & 2478058128 & M \\
\hline & 40 & 6 & 23410 & 1 & 1769570 & 6051 & 1225250 & 476 & 14455339080 & M \\
\hline & 40 & 7 & 23410 & 1 & 1769570 & 5282 & 1225250 & 467 & 70211646960 & M \\
\hline & 50 & 1 & 29220 & 1 & 2754420 & 6316 & 1906020 & 1031 & 225100 & 14 \\
\hline & 50 & 2 & 29220 & 1 & 2754420 & 13331 & 1906020 & 1818 & 5514950 & 98 \\
\hline & 50 & 3 & 29220 & 1 & 2754420 & 15007 & 1906020 & 2848 & 88239200 & M \\
\hline & 50 & 4 & 29220 & 1 & 2754420 & 17181 & 1906020 & 3225 & 1036810600 & M \\
\hline & 50 & 5 & 29220 & 1 & 2754420 & 19075 & 1906020 & 3413 & 9538657520 & M \\
\hline & 50 & 6 & 29220 & 1 & 2754420 & 12814 & 1906020 & 2867 & 71539931400 & M \\
\hline & 50 & 7 & 29220 & 1 & 2754420 & 11557 & 1906020 & 2333 & 449679568800 & M \\
\hline & 10 & 1 & 7278 & 1 & 142278 & 5 & 99438 & 3 & 13020 & 1 \\
\hline & 10 & 2 & 7278 & 1 & 142278 & 5 & 99438 & 3 & 58590 & 5 \\
\hline & 10 & 3 & 7278 & 1 & 142278 & 4 & 99438 & 4 & 156240 & 1 \\
\hline & 10 & 4 & 7278 & 1 & 142278 & 4 & 99438 & 2 & 273420 & 2 \\
\hline & 10 & 5 & 7278 & 1 & 142278 & 3 & 99438 & 2 & 328104 & 2 \\
\hline & 10 & 6 & 7278 & 1 & 142278 & 4 & 99438 & 3 & 273420 & 2 \\
\hline & 10 & 7 & 7278 & 1 & 142278 & 3 & 99438 & 2 & 156240 & 1 \\
\hline & 20 & 1 & 14338 & 1 & 547938 & 99 & 380658 & 33 & 32040 & 1 \\
\hline & 20 & 2 & 14338 & 1 & 547938 & 100 & 380658 & 68 & 304380 & 5 \\
\hline & 20 & 3 & 14338 & 1 & 547938 & 134 & 380658 & 61 & 1826280 & 47 \\
\hline & 20 & 4 & 14338 & 1 & 547938 & 80 & 380658 & 59 & 7761690 & 292 \\
\hline & 20 & 5 & 14338 & 1 & 547938 & 59 & 380658 & 67 & 24837408 & M \\
\hline & 20 & 6 & 14338 & 1 & 547938 & 83 & 380658 & 38 & 62093520 & M \\
\hline & 20 & 7 & 14338 & 1 & 547938 & 59 & 380658 & 50 & 124187040 & M \\
\hline & 30 & 1 & 21404 & 1 & 1217384 & 627 & 844064 & 147 & 57000 & 4 \\
\hline & 30 & 2 & 21404 & 1 & 1217384 & 677 & 844064 & 173 & 826500 & 14 \\
\hline & 30 & 3 & 21404 & 1 & 1217384 & 722 & 844064 & 192 & 7714000 & 229 \\
\hline
\end{tabular}




\begin{tabular}{|c|c|c|c|c|c|c|c|c|c|c|}
\hline & 30 & 4 & 21404 & 1 & 1217384 & 777 & 844064 & 175 & 52069500 & M \\
\hline & 30 & 5 & 21404 & 1 & 1217384 & 741 & 844064 & 286 & 270761400 & M \\
\hline & 30 & 6 & 21404 & 1 & 1217384 & 603 & 844064 & 205 & 1128172500 & M \\
\hline & 30 & 7 & 21404 & 1 & 1217384 & 493 & 844064 & 153 & 3868020000 & M \\
\hline & 40 & 1 & 28474 & 1 & 2150714 & 2480 & 1489754 & 470 & 76000 & 1 \\
\hline & 40 & 2 & 28474 & 1 & 2150714 & 2796 & 1489754 & 512 & 1482000 & 1 \\
\hline & 40 & 3 & 28474 & 1 & 2150714 & 2613 & 1489754 & 612 & 18772000 & 762 \\
\hline & 40 & 4 & 28474 & 1 & 2150714 & 2866 & 1489754 & 591 & 173641000 & M \\
\hline & 40 & 5 & 28474 & 1 & 2150714 & 3251 & 1489754 & 588 & 1250215200 & M \\
\hline & 40 & 6 & 28474 & 1 & 2150714 & 3187 & 1489754 & 757 & 7292922000 & M \\
\hline & 40 & 7 & 28474 & 1 & 2150714 & 2594 & 1489754 & 885 & 35422764000 & M \\
\hline & 50 & 1 & 35550 & 1 & 3348150 & 28000 & 2317950 & 1212 & 95000 & 1 \\
\hline & 50 & 2 & 35550 & 1 & 3348150 & 28000 & 2317950 & 1381 & 2327500 & 56 \\
\hline & 50 & 3 & 35550 & 1 & 3348150 & 28000 & 2317950 & 1525 & 37240000 & M \\
\hline & 50 & 4 & 35550 & 1 & 3348150 & 28000 & 2317950 & 1711 & 437570000 & M \\
\hline & 50 & 5 & 35550 & 1 & 3348150 & 28000 & 2317950 & 1796 & 4025644000 & M \\
\hline & 50 & 6 & 35550 & 1 & 3348150 & 28000 & 2317950 & 2133 & 30192330000 & M \\
\hline & 50 & 7 & 35550 & 1 & 3348150 & 28000 & 2317950 & 2369 & 189780360000 & M \\
\hline & 10 & 1 & 12640 & 1 & 240520 & 9 & 168280 & 7 & 30500 & 1 \\
\hline & 10 & 2 & 12640 & 1 & 240520 & 9 & 168280 & 6 & 137250 & 12 \\
\hline & 10 & 3 & 12640 & 1 & 240520 & 6 & 168280 & 6 & 366000 & 3 \\
\hline & 10 & 4 & 12640 & 1 & 240520 & 6 & 168280 & 7 & 640500 & 6 \\
\hline & 10 & 5 & 12640 & 1 & 240520 & 5 & 168280 & 6 & 768600 & 5 \\
\hline & 10 & 6 & 12640 & 1 & 240520 & 5 & 168280 & 6 & 640500 & 4 \\
\hline & 10 & 7 & 12640 & 1 & 240520 & 4 & 168280 & 8 & 366000 & 2 \\
\hline & 20 & 1 & 24594 & 1 & 925834 & 144 & 643754 & 91 & 71200 & 3 \\
\hline & 20 & 2 & 24594 & 1 & 925834 & 185 & 643754 & 84 & 676400 & 6 \\
\hline & 20 & 3 & 24594 & 1 & 925834 & 157 & 643754 & 111 & 4058400 & 98 \\
\hline & 20 & 4 & 24594 & 1 & 925834 & 182 & 643754 & 147 & 17248200 & 717 \\
\hline & 20 & 5 & 24594 & 1 & 925834 & 183 & 643754 & 95 & 55194240 & M \\
\hline & 20 & 6 & 24594 & 1 & 925834 & 161 & 643754 & 118 & 137985600 & M \\
\hline & 20 & 7 & 24594 & 1 & 925834 & 145 & 643754 & 87 & 275971200 & M \\
\hline & 30 & 1 & 36564 & 1 & 2057124 & 1987 & 1427604 & 574 & 152520 & 15 \\
\hline & 30 & 2 & 36564 & 1 & 2057124 & 1910 & 1427604 & 504 & 2211540 & 36 \\
\hline & 30 & 3 & 36564 & 1 & 2057124 & 2160 & 1427604 & 539 & 20641040 & 942 \\
\hline & 30 & 4 & 36564 & 1 & 2057124 & 1649 & 1427604 & 513 & 139327020 & M \\
\hline & 30 & 5 & 36564 & 1 & 2057124 & 2619 & 1427604 & 463 & 724500504 & M \\
\hline & 30 & 6 & 36564 & 1 & 2057124 & 1845 & 1427604 & 630 & 3018752100 & M \\
\hline & 30 & 7 & 36564 & 1 & 2057124 & 1076 & 1427604 & 652 & 10350007200 & 1 \\
\hline
\end{tabular}




\begin{tabular}{rrrrrrrrrr}
40 & 1 & 48524 & 1 & 3633804 & 28000 & 2519244 & 1791 & 203360 & 36 \\
40 & 2 & 48524 & 1 & 3633804 & 28000 & 2519244 & 1961 & 3965520 & 82 \\
40 & 3 & 48524 & 1 & 3633804 & 28000 & 2519244 & 2254 & 50229920 & $\mathrm{M}$ \\
40 & 4 & 48524 & 1 & 3633804 & 28000 & 2519244 & 2441 & 464626760 & $\mathrm{M}$ \\
40 & 5 & 48524 & 1 & 3633804 & 28000 & 2519244 & 2054 & 3345312672 & $\mathrm{M}$ \\
40 & 6 & 48524 & 1 & 3633804 & 28000 & 2519244 & 2657 & 19514323920 & $\mathrm{M}$ \\
40 & 7 & 48524 & 1 & 3633804 & 28000 & 2519244 & 2155 & 94783859040 & $\mathrm{M}$ \\
50 & 1 & 60478 & 1 & 5655778 & $\mathrm{M}$ & 3918578 & 4536 & 279500 & 61 \\
50 & 2 & 60478 & 1 & 5655778 & $\mathrm{M}$ & 3918578 & 5188 & 6847750 & 217 \\
50 & 3 & 60478 & 1 & 5655778 & $\mathrm{M}$ & 3918578 & 5360 & 109564000 & $\mathrm{M}$ \\
50 & 4 & 60478 & 1 & 5655778 & $\mathrm{M}$ & 3918578 & 6858 & 1287377000 & $\mathrm{M}$ \\
50 & 5 & 60478 & 1 & 5655778 & $\mathrm{M}$ & 3918578 & 4959 & 11843868400 & $\mathrm{M}$ \\
50 & 6 & 60478 & 1 & 5655778 & $\mathrm{M}$ & 3918578 & 5044 & 88829013000 & $\mathrm{M}$ \\
50 & 7 & 60478 & 1 & 5655778 & $\mathrm{M}$ & 3918578 & 4992 & 558353796000 & $\mathrm{M}$ \\
\hline
\end{tabular}

Table 3: Comparing static, affine, and dynamic routing in terms of computational time.

\section{Concluding Remarks}

In this paper we study two-stage robust network design problems with affine recourse for flow. We show that the resulting affine routing provides a reasonable alternative to the well-studied static and dynamic routing schemes. All three schemes are embedded into the general framework of two-stage network design with recourse.

Similar to the static case, the affine robust counterpart is tractable but it turns out computationally to provide solutions as cheap as the dynamic models.

We investigated the three routing principles with respect to their flexibility depending on the structure of the given demand uncertainty set $\mathcal{D}$. Fixing the demand polytope $\mathcal{D}$, the cost of optimal affine solutions is always between the cost for optimal static and optimal dynamic solutions. In this work we develop necessary and sufficient conditions on $\mathcal{D}$ under which affine routings reduce to static routings and also develop properties of uncertainty sets leading to dynamic routings being affine.

We also consider the well-known concept of domination between demand-vectors and show that in contrast to the static and dynamic case there is no affine domination. That is, given two demand-vectors, there is always an affine solution feasible for one but not for the other. In this respect affine routings suffer from the drawback that even totally dominated demand vectors are 
not necessarily supported by affine solutions. Uncertainty sets have to be designed accordingly in practice.

Finally, we compute the cost gap between static, affine, and dynamic solutions based on networks from SNDlib and the uncertainty model studied by Bertsimas and Sim. We conclude that for these instances the solutions based on affine routings tend to be as cheap as two-stage solutions with dynamic recourse. In this respect the affine routing principle allows enough flexibility to almost capture dynamic routings. Since it is in general NP-hard to compute an optimal network design with dynamic routing, the affine principle can hence be used to approximate free recourse using tractable robust counterparts.

Affine models turn out to be attractive since polynomial reformulations are available. These formulations however tend to be very large, so they become hard to solve for large instances. For practical purposes one has to work on the formulations and methods to solve affine problems. In this context it might be wise to restrict the number of commodities in the affine recourse and to apply decomposition methods.

\section{References}

[1] A. Altin, E. Amaldi, P. Belotti, and M. Ç. Pinar, Virtual private network design under traffic uncertainty, Electronic Notes in Discr Math 17 (2004), 19-22.

[2] A. Altin, E. Amaldi, P. Belotti, and M. Ç. Pinar, Provisioning virtual private networks under traffic uncertainty, Networks 49 (2007), 100-115.

[3] A. Altin, H. Yaman, and M. Pinar, "A hybrid polyhedral uncertainty model for the robust network loading problem," Performance models and risk management in communications systems, N. Gülpinar, P. Harrison, and B. Rustem (Editors), Springer, 2011, pp. 157-172.

[4] A. Altin, H. Yaman, and M. Pinar, The robust network loading problem under hose demand uncertainty: Formulation, polyhedral analysis, and computations, INFORMS J Comput 23 (2011), 75-89.

[5] D. Applegate and E. Cohen, Making intra-domain routing robust to changing and uncertain traffic demands: Understanding fundamental tradeoffs, Proc Conference Appl, Technologies, Architectures, Protocols Comput Commun (SIGCOM 2003), Karlsruhe, Germany, 2003, pp. 313-324.

[6] A. Atamturk, On capacitated network design cut-set polyhedra, Math Program 92 (2002), 425-437. 
[7] F. Babonneau, O. Klopfenstein, A. Ouorou, and J.P. Vial, Robust capacity expansion solutions for telecommunication networks with uncertain demands, Networks (submitted, available as Optimization Online preprint 2712).

[8] W. Ben-Ameur, Between fully dynamic routing and robust stable routing, Proc 6th Int Workshop Design Reliable Commun Networks (DRCN 2007), 2007, pp. 1-6.

[9] W. Ben-Ameur and H. Kerivin, New economical virtual private networks, Commun ACM 46 (2003), 69-73.

[10] W. Ben-Ameur and H. Kerivin, Routing of uncertain demands, Optim Eng 3 (2005), 283-313.

[11] A. Ben-Tal, A. Goryashko, E. Guslitzer, and A. Nemirovski, Adjustable robust solutions of uncertain linear programs, Math Program 99 (2004), 351-376.

[12] A. Ben-Tal and A. Nemirovski, Robust solutions of uncertain linear programs, Oper Res Lett 25 (1999), 1-13.

[13] A. Ben-Tal and A. Nemirovski, Robust solutions of linear programming problems contaminated with uncertain data, Math Program 88 (2000), 411-424.

[14] A. Ben-Tal and A. Nemirovski, Robust optimization methodology and applications, Math Program 92 (2002), 453-480.

[15] J. Benders, Partitioning procedures for solving mixed-variables programming problems, $\mathrm{Nu}-$ mer Mathematik 4 (1962), 238-252.

[16] D. Bertsimas and V. Goyal, On the power and limitations of affine policies in two-stage adaptive optimization, Math Program (2011, Online First).

[17] D. Bertsimas and M. Sim, Robust discrete optimization and network flows, Math Program 98 (2003), 49-71.

[18] D. Bertsimas and M. Sim, The price of robustness, Oper Res 52 (2004), 35-53.

[19] D. Bienstock and O. Günlück, Capacitated network design - Polyhedral structure and computation, INFORMS J Comput 8 (1996), 243-259.

[20] A. Bley, R. Klaehne, U. Menne, C. Raack, and R. Wessaely, Multi-layer network design - A model-based optimization approach, Proc PGTS 2008, Berlin, Germany, 2008, pp. 107-116.

[21] C. Chekuri, Routing and network design with robustness to changing or uncertain traffic demands, ACM SIGACT News 38 (2007), 106-129. 
[22] C. Chekuri, G. Oriolo, M.G. Scutellà, and F.B. Shepherd, Hardness of robust network design, Networks 50 (2007), 50-54.

[23] X. Chen and Y. Zhang, Uncertain linear programs: Extended affinely adjustable robust counterparts, Oper Res 57 (2009), 1469-1482.

[24] A.M. Costa, A survey on Benders decomposition applied to fixed-charge network design problems, Comput. Oper Res 32 (2005), 1429-1450.

[25] G. Dahl and M. Stoer, A cutting plane algorithm for multicommodity survivable network design problems, INFORMS J. Comput 10 (1998), 1-11.

[26] N.G. Duffield, P. Goyal, A. Greenberg, P. Mishra, K.K. Ramakrishnan, and J.E. van der Merive, A flexible model for resource management in virtual private networks, SIGCOMM Comput. Commun. Rev. 29 (1999), 95-108.

[27] A. Dwivedi and R.E. Wagner, Traffic model for USA long-distance optical network, Proc Optical Fiber Commun Conference (OFC 2000), 2000, pp. 156-158.

[28] A. Frangioni and B. Gendron, 0-1 reformulations of the multicommodity capacitated network design problem, Discr Appl Math 157 (2009), 1229-1241.

[29] A. Frangioni, F. Pascali, and M.G. Scutellà, Static and dynamic routing under disjoint dominant extreme demands, Oper Res Lett 39 (2011), 36-39.

[30] N. Goyal, N. Olver, and F.B. Shepherd, Dynamic vs. oblivious routing in network design, Proc ESA 2009, 2009, pp. 277-288.

[31] A. Gupta, J. Kleinberg, A. Kumar, R. Rastogi, and B. Yener, Provisioning a virtual private network: A network design problem for multicommodity flow, Proc 33rd annual ACM Symp Theory Comput (STOC 2001), Heraklion, Crete, Greece, 2001, pp. 389-398.

[32] IBM, IBM-ILOG Cplex Optimizer, webpage. http://www-01.ibm.com/software/ integration/optimization/cplex-optimizer/.

[33] M. Iri, On an extension of the maximum-flow minimum-cut theorem to multicommodity flows, J Oper Res Soc Jpn 13 (1971), 129-135.

[34] T. Koch, Zimpl user guide, Technical report ZR 01-20, Zuse Institute Berlin, 2001.

[35] A.M.C.A. Koster, M. Kutschka, and C. Raack, Cutset inequalities for robust network design, Proc 5th Int Network Optim Conference (INOC 2011), Hamburg, Germany, Network Optimization - Lecture Notes in Computer Science, Vol. 6701, Springer, 2011, pp. 118-123. 
[36] A.M.C.A. Koster, M. Kutschka, and C. Raack, Robust network design: Formulations, valid inequalities, and computations, ZIB report 11-34, Zuse Institute Berlin, 2011.

[37] A. Lisser, A. Ouorou, J.P. Vial, and J. Gondzio, Capacity planning under uncertain demand in telecommunication networks, Papers 99.13, Ecole des Hautes Etudes Commerciales, Universite de Geneve, 1999.

[38] S. Mattia, The robust network loading problem with dynamic routing, Technical report n. 3, La Sapienza, University of Rome, 2010.

[39] S. Mudchanatongsuk, F. Ordonez, and J. Liu, Robust solutions for network design under transportation cost and demand uncertainty, J Oper Res Soc 59 (2008), 552-562.

[40] K. Onaga and O. Kakusho, On feasibility conditions of multicommodity flows in networks, Trans Circuit Theory 18 (1971), 425-429.

[41] F. Ordóñez and J. Zhao, Robust capacity expansion of network flows, Networks 50 (2007), $136-145$.

[42] G. Oriolo, Domination between traffic matrices, Math Oper Res 33 (2008), 91-96.

[43] S. Orlowski, M. Pióro, A. Tomaszewski, and R. Wessäly, SNDlib 1.0-Survivable Network Design Library, Networks 55 (2010), 276-286, http://sndlib.zib.de.

[44] A. Ouorou, Affine decision rules for tractable approximations to robust capacity planning in telecommunications, Proc 5th Int Network Optim Conference (INOC 2011), Hamburg, Germany, Network Optimization - Lecture Notes in Computer Science, Vol. 6701, Springer, 2011, pp. 277-282.

[45] A. Ouorou and J.P. Vial, A model for robust capacity planning for telecommunications networks under demand uncertainty, Proc 6th Int Workshop Design Reliable Commun Networks (DRCN 2007), 2007, pp. 1-4.

[46] M. Poss and C. Raack, Affine recourse for the robust network design problem: Between static and dynamic routing, Proc 5th Int Network Optim Conference (INOC 2011), Hamburg, Germany, Network Optimization - Lecture Notes in Computer Science, Vol. 6701, Springer, 2011, pp. 150-155.

[47] C. Raack, A.M.C.A. Koster, S. Orlowski, and R. Wessäly, On cut-based inequalities for capacitated network design polyhedra, Networks 57 (2011), 141-156.

[48] M.G. Scutellà, On improving optimal oblivious routing, Oper Res Lett 37 (2009), 197-200. 
[49] M.G. Scutellà, Hardness of some optimal oblivious routing generalizations, Technical report TR-10-05, Universitá di Pisa, 2010.

[50] A.L. Soyster, Convex programming with set-inclusive constraints and applications to inexact linear programming, Oper Res 21 (1973), 1154-1157.

[51] Y. Zhang, M. Roughan, N. Duffield, and A. Greenberg, Fast accurate computation of largescale IP traffic matrices from link loads, Proc ACM SIGMETRICS, 2003, pp. 206-217. 